\title{
SHELLABLE NONPURE COMPLEXES AND POSETS. I
}

\author{
ANDERS BJÖRNER AND MICHELLE L. WACHS
}

\begin{abstract}
The concept of shellability of complexes is generalized by deleting the requirement of purity (i.e., that all maximal faces have the same dimension). The usefulness of this level of generality was suggested by certain examples coming from the theory of subspace arrangements. We develop several of the basic properties of the concept of nonpure shellability.

Doubly indexed $f$-vectors and $h$-vectors are introduced, and the latter are shown to be nonnegative in the shellable case. Shellable complexes have the homotopy type of a wedge of spheres of various dimensions, and their StanleyReisner rings admit a combinatorially induced direct sum decomposition.

The technique of lexicographic shellability for posets is similarly extended from pure posets (all maximal chains of the same length) to the general case. Several examples of nonpure lexicographically shellable posets are given, such as the $k$-equal partition lattice (the intersection lattice of the $k$-equal subspace arrangement) and the Tamari lattices of binary trees. This leads to simplified computation of Betti numbers for the $k$-equal arrangement. It also determines the homotopy type of intervals in a Tamari lattice and in the lattice of number partitions ordered by dominance, thus strengthening some known Möbius function formulas.

The extension to regular CW complexes is briefly discussed and shown to be related to the concept of lexicographic shellability.
\end{abstract}

\section{CONTENTS}

\section{Part I}

1. Introduction

2. Shellable simplicial complexes

3. Face numbers and the $h$-triangle

4. Topological properties

5. Lexicographically shellable posets

6 . The $k$-equal partition lattice

7. Other posets of set partitions

Part II

8. Interval-generated lattices and dominance order

9. The Tamari lattices

10. Constructions that preserve shellability

11. Shifting, vertex-decomposability and CL-shellability

Received by the editors October 26, 1994.

1991 Mathematics Subject Classification. Primary 05E99, 06A08; Secondary 52B20, 55U15, $57 \mathrm{Q} 05$.

Research of the second author partially supported by NSF grants DMS 9102760 and DMS 9311805 . 
12. Decomposition of the Stanley-Reisner ring

13. Regular cell complexes

\section{INTRODUCTION}

A simplicial complex is said to be pure if all facets (i.e., maximal faces) are of equal dimension. According to the standard definition a complex is shellable if it is pure and its facets can be ordered so that each one (other than the first) intersects the union of its predecessors in a nonempty union of maximal proper faces. Shellability is a well-known concept in combinatorics and combinatorial topology and geometry with several useful consequences of algebraic, combinatorial and topological nature.

In this paper we drop the requirement of purity from the definition and explore a more general concept of shellability. Most of the properties known from the pure case generalize, although the combinatorial analysis is in some parts more involved and there are some new phenomena that provide for a richer theory.

We were led to consider shellability for nonpure complexes in a search for combinatorial tools to analyze intersection lattices of subspace arrangements. The first example obtained, the intersection lattice of the $k$-equal subspace arrangement (Section 6), convinced us that the general concept is worth developing. In Sections 7-9 we present several other nontrivial examples of nonpure shellability. Further examples are likely to be found for instance among posets of subgroups of a finite group, see the survey of Welker [We] for the role of pure shellability in that area.

The rest of this introduction will consist of sectionwise descriptions of content and additional comments.

Sections 8-13 are contained in PartII, which will appear in a later issue of this journal. Section 2 deals with some elementary combinatorial properties of shellability, closely related to the definition. There are different ways to view the concept and these lead to different characterizations.

In Section 3 we consider the enumeration of faces of nonpure complexes. Doubly indexed numbers $f_{i, j}$ are defined that refine the usual $f$-vector. There are corresponding $h_{i, j}$ numbers, parallel to the usual $h$-vector, and these are shown to be nonnegative in the shellable case.

The topological properties of shellable complexes are treated in Section 4. The basic fact here is that a shellable complex has the homotopy type of a wedge of spheres (of various dimensions), and the diagonal $h$-numbers $h_{i, i}$ give the Betti numbers. We also show how bases for homology and for cohomology are combinatorially induced.

Section 5 develops the theory of lexicographic shellability for nonpure posets. This is a method to obtain the shellability of the order complex of a poset by labeling the edges of its Hasse diagram subject to some simple combinatorial rules. In a weaker version ( $R$-labelings) one obtains a formula for the Möbius function. The task of computing Betti numbers for a lexicographically shellable poset is reduced to that of counting maximal chains with nonincreasing label sequence. Lexicographic shellability has a useful alternative version as a recursively defined ordering of the atoms of a poset. Several examples of nonpure lexicographically shellable posets are given in Sections 6-9.

The $k$-equal partition lattices consist of partitions of $\{1, \ldots, n\}$ with all blocks of size 1 or of size $\geq k$. Alternatively it is the lattice of intersections of the subspaces 
in $\mathbb{R}^{n}$ defined by setting $k$ coordinates equal. We show that it is lexicographically shellable in Section 6 and derive formulas for its Betti numbers, one previously known from $[\mathrm{BWe}]$ and one new.

In Section 7 we consider a number of other lattices of set partitions, related to other subspace arrangements and to hypergraphs. We show that some are shellable and others not.

Section 8 deals with lattices of sets that arise as unions of members from some given family of integer intervals. We show that a natural class of such lattices are lexicographically shellable. Using an idea of Greene $[\mathrm{G}]$ we can from this transfer topological information to the lattice $P_{n}$ of all number partitions of the integer $n$ ordered by dominance. Intervals in $P_{n}$ are shown to be either contractible or homotopy spheres.

The Tamari lattices $T_{n}$, discussed in Section 9, are also called rotation lattices of binary trees. We show that they are lexicographically shellable, from which we deduce that all intervals are either contractible or homotopy spheres. For both $T_{n}$ and the dominance lattice $P_{n}$ these topological facts strengthen known results about their Möbius function. In a somewhat different direction we show that $T_{n}$ is induced on the set of binary trees as a quotient of weak order of the symmetric group $S_{n}$. Also, the set of 312 -free permutations is shown to form a sublattice of weak order which is isomorphic to $T_{n}$.

In Section 10 we discuss various ways that shellability is preserved under operations on complexes and posets. It is one of the useful properties of shellability, somewhat surprising in view of its rigid definition, that the concept is so flexible and adaptable. We spend some time discussing "nonpure rank-selection", i.e., how shellability is inherited by various vertex-induced subcomplexes and subposets.

Section 11 continues the discussion of technical properties of nonpure shellable complexes and posets. The class of nonpure vertex-decomposable complexes is introduced, extending a definition of Provan and Billera $[\mathrm{PB}]$ from the pure case. It is shown that "shifted" $\Longrightarrow$ "vertex-decomposable" $\Longrightarrow$ "shellable", and that "CL-shellable" $\Longrightarrow$ "vertex-decomposable". The analysis reveals some special dismantling properties of CL-shellable posets.

In the pure case the notion of shellability implies Cohen-Macaulayness of the Stanley-Reisner ring of a complex, and this connection has given much (but certainly not all) of the motivation for studying pure shellability. In its most combinatorial version this implication takes the form of a direct sum decomposition of the ring, which in the pure case was discovered by Garsia [Ga] and Kind and Kleinschmidt $[\mathrm{KK}]$. In Section 12 we prove the corresponding type of decomposition for nonpure shellable complexes.

Some of the theory of nonpure shellability extends to regular CW complexes, and for this we are able to partially fall back on lexicographic shellability via face lattices. This material is briefly outlined in Section 13 .

The basic results of this paper (Sections 2-6) were obtained in November 1992, and the rest of the paper was also drafted at that time. For various reasons it has taken us almost two years to get it into writing. In the meanwhile we have presented these results orally in several places, which has led to some interesting further developments. Sundaram and Wachs $[\mathrm{SW}]$ have used the lexicographic shellability of the $k$-equal partition lattice (the actual labeling plays a fundamental role) to determine the representations of the symmetric group on its homology. Building 
on this, Sundaram and Welker [SWe] have obtained the representations of $S_{n}$ on the cohomology of the complement of the $k$-equal arrangement. The intersection lattices of $k$-equal subspace arrangements of type $B_{n}$ were shown to be lexicographically shellable by Björner and Sagan [BS], which was then used to compute their homology. Linusson [L1], Sundaram [Su] and Sanders and Wachs [SaW] have studied the partition lattices $\Pi_{n, k}^{\prime}$ of Section 7 and other posets defined by forbidden block sizes. The Möbius function of $\Pi_{n, k}^{\prime}$ is applied to a complexity problem in [L1], the virtual representation of $S_{n}$ induced on the alternating sum of its homology is determined in [Su], and bases for homology and cohomology together with representation-theoretic consequences are given in $[\mathrm{SaW}]$. The topological results about interval-generated lattices in Section 8 have been generalized by Linusson [L2]. Stanley [S5] has introduced the concept of "sequential Cohen-Macaulayness" as an algebraic counterpart (for the Stanley-Reisner ring) to nonpure shellability. Finally, Björner [B7] has used nonpure shellability as a part of proving a characterization of $f$-vectors for complexes whose Stanley-Reisner ring has depth $\geq k$.

\section{Shellable Simplicial COMPleXes}

We begin with some basic definitions. A complex (or abstract simplicial complex) $\Delta$ on a vertex set $V$ is a finite collection of subsets of $V$, called faces, such that $A \subseteq B \in \Delta$ implies $A \in \Delta$. Dimension is defined by $\operatorname{dim} A=|A|-1$ and $\operatorname{dim} \Delta=\max \operatorname{dim} A$. The prefix " $k$-" in $k$-face and $k$-complex denotes dimension. The empty set is a face of every nonempty complex. We allow also the degenerate $(-1)$-dimensional complex $\{\emptyset\}$.

For sets $A \subseteq B$ we have the Boolean interval $[A, B]=\{C \mid A \subseteq C \subseteq B\}$. Let $\bar{A}=[\emptyset, A]$. A complex of the form $\bar{A}$ is called a simplex.

The inclusionwise maximal faces of a complex are called facets. A complex is pure if all facets are of equal dimension.

2.1. Definition. A complex $\Delta$ is shellable if its facets can be arranged in linear order $F_{1}, F_{2}, \ldots, F_{t}$ in such a way that the subcomplex $\left(\bigcup_{i=1}^{k-1} \bar{F}_{i}\right) \cap \bar{F}_{k}$ is pure and ( $\left.\operatorname{dim} F_{k}-1\right)$-dimensional for all $k=2, \ldots, t$. Such an ordering of facets is called a shelling .

The idea with this definition is that $\Delta$ can be built stepwise by introducing the facets one at a time and attaching each new facet $F_{k}$ to the complex previously built in the nicest possible fashion. See Figure 1 for examples of shellable and nonshellable complexes.

2.2. Lemma. If $F_{1}, F_{2}, \ldots, F_{t}$ is a shelling of $\Delta$, then $\operatorname{dim} F_{1}=\operatorname{dim} \Delta$.

Proof. If $\operatorname{dim} F_{i}<\operatorname{dim} F_{k}$ for all $1 \leq i<k$, then $\operatorname{dim}\left[\left(\bigcup_{i=1}^{k-1} \bar{F}_{i}\right) \cap \bar{F}_{k}\right] \leq$ $\operatorname{dim} F_{k}-2$.

The following is a straightforward restatement of Definition 2.1, that is often useful.

2.3. Lemma. An order $F_{1}, F_{2}, \ldots, F_{t}$ of the facets of $\Delta$ is a shelling if and only if for every $i$ and $k$ with $1 \leq i<k \leq t$ there is a $j$ with $1 \leq j<k$ and an $x \in F_{k}$ such that $F_{i} \cap F_{k} \subseteq F_{j} \cap F_{k}=F_{k}-\{x\}$.

There is another reformulation of the concept of shellability (Proposition 2.5) that brings out a very important partitioning property. We have chosen to define 
<smiles>OO</smiles>

(a)

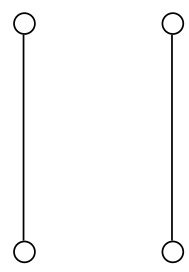

(b)

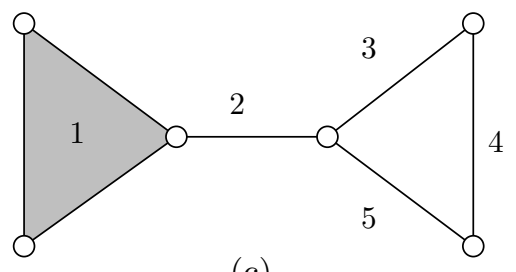

(c)

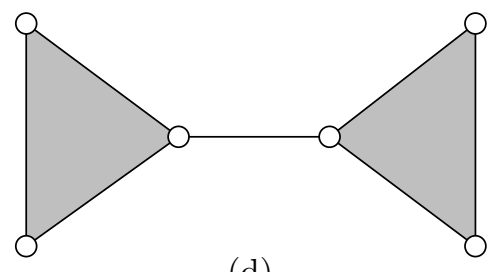

(d)

Shellable

Not shellable

FIGURE 1

the concept as was done in Definition 2.1 rather than in terms of partitioning, because this is the geometric version of the idea that generalizes to cell complexes, see Section 13 for this.

2.4. Lemma. Let $F$ be a facet of a complex $\Delta, R \subseteq F$, and let $\Delta^{\prime}$ be the subcomplex generated by the other facets of $\Delta$. Then the following statements are equivalent:

(1) $\bar{F}-\Delta^{\prime}=[R, F]$,

(2) $\bar{F} \cap \Delta^{\prime}=\bigcup_{x \in R} \overline{F-\{x\}}$.

Proof. $\bar{F}$ is clearly the disjoint union of $[R, F]$ and $\bigcup_{x \in R} \overline{F-\{x\}}$.

Given a shelling $F_{1}, F_{2}, \ldots, F_{t}$ of $\Delta$, with successively generated subcomplexes $\Delta_{j}=\bigcup_{i=1}^{j} \bar{F}_{i}$, define the restriction of facet $F_{k}$ by

$$
\mathcal{R}\left(F_{k}\right)=\left\{x \in F_{k} \mid F_{k}-\{x\} \in \Delta_{k-1}\right\}
$$

Lemma 2.4 shows that $\bar{F}_{k}-\Delta_{k-1}=\left[\mathcal{R}\left(F_{k}\right), F_{k}\right]$, in other words: $\mathcal{R}\left(F_{k}\right)$ is the unique minimal new face introduced in the $k$-th shelling step. Hence, when adding the new facet $F_{k}$ to $\Delta_{k-1}$ we in fact add a Boolean interval of new faces, so by induction $\Delta$ splits up into a disjoint union of Boolean intervals:

$$
\Delta=\bigcup_{i=1}^{t}\left[\mathcal{R}\left(F_{i}\right), F_{i}\right]
$$

Shellability can be characterized in terms of such decompositions. 
2.5. Proposition. Given an ordering $F_{1}, \ldots, F_{t}$ of the facets of $\Delta$ and a map $\mathcal{R}:\left\{F_{1}, \ldots, F_{t}\right\} \rightarrow \Delta$, the following are equivalent:

(1) $F_{1}, \ldots, F_{t}$ is a shelling and $\mathcal{R}$ its restriction map,

(2) $\begin{cases}(\alpha) & \Delta=\dot{\bigcup}_{i=1}^{t}\left[\mathcal{R}\left(F_{i}\right), F_{i}\right], \text { and } \\ (\beta) & \mathcal{R}\left(F_{i}\right) \subseteq F_{j} \text { implies } i \leq j, \text { for all } i, j .\end{cases}$

Proof. The implication $(1) \Rightarrow(2)$ is already proved. The opposite implication follows from Lemma 2.4 .

The first facet of a shelling is always of maximal dimension, as was shown in Lemma 2.2. In fact, if $\Delta$ is shellable there is always a shelling in which the facets appear in order of decreasing dimension.

2.6. Rearrangement lemma. Let $F_{1}, F_{2}, \ldots, F_{t}$ be a shelling of $\Delta$ with restriction map $\mathcal{R}$. Let $F_{i_{1}}, F_{i_{2}}, \ldots, F_{i_{t}}$ be the rearrangement obtained by taking first all facets of dimension $d-1=\operatorname{dim} \Delta$ in the induced order, then all facets of dimension $d-2$ in the induced order, and continuing this way in order of decreasing dimension. Then this rearrangement is also a shelling, and its restriction map $\mathcal{R}^{\prime}$ is the same: $\mathcal{R}^{\prime}(F)=\mathcal{R}(F)$ for all facets $F$.

Proof. Using Proposition 2.5 one sees that it suffices to verify that

$$
\mathcal{R}\left(F_{i_{j}}\right) \subseteq F_{i_{k}} \Longrightarrow j \leq k .
$$

If $(*)$ is violated, there must exist indices $a$ and $b$ such that

$$
a<b,\left|F_{a}\right|<\left|F_{b}\right| \text {, and } \mathcal{R}\left(F_{a}\right) \subseteq F_{b} .
$$

Suppose that $(*)$ fails, and choose $a$ and $b$ with $b$ minimal in $(* *)$. We have that $\mathcal{R}\left(F_{a}\right) \neq F_{b}$, since $F_{b} \nsubseteq F_{a}$. Then $\mathcal{R}\left(F_{a}\right) \subseteq A \subset F_{b}$ for some $A=F_{b}-\{x\}$, and from the shelling property of $F_{1}, \ldots, F_{t}$ it follows that $A \subset F_{c}$ for some $c<b$. Then $\mathcal{R}\left(F_{a}\right) \subset F_{c}$, so $a<c$, and also $\left|F_{c}\right| \geq\left|F_{b}\right|$. Hence there is a violation of the choice of $b$ as being minimal in $(* *)$.

2.7. Second rearrangement lemma. Let $F_{1}, F_{2}, \ldots, F_{t}$ be a shelling of $\Delta$ with restriction map $\mathcal{R}$. Let $F_{i_{1}}, F_{i_{2}}, \ldots, F_{i_{t}}$ be the rearrangement obtained by taking first all facets $F$ such that $\mathcal{R}(F) \neq F$ in the induced order, and then all remaining facets in arbitrary order. Then this rearrangement is also a shelling with the same restriction map $\mathcal{R}$.

Proof. Immediate from either Definition 2.1 or Proposition 2.5.

2.8. Definition. Let $\Delta$ be a complex, and let $0 \leq r \leq s \leq \operatorname{dim} \Delta$.

(1) $\Delta$ is $(r, s)$-pure if $r \leq \operatorname{dim} F \leq s$ for all facets $F$.

(2) $\Delta^{(r, s)}=\{A \in \Delta \mid \operatorname{dim} A \leq s$ and $A \subseteq F$ for some facet $F$ with $\operatorname{dim} F \geq r\}$.

For example, $\Delta^{(0, s)}$ is the $s$-skeleton of $\Delta$, and $\Delta^{(s, s)}$ is the subcomplex of $\Delta$ generated by all $s$-faces. Clearly, $\Delta^{(r, s)}$ is $(r, s)$-pure.

2.9. Theorem. If $\Delta$ is shellable, then so is $\Delta^{(r, s)}$, for all $r \leq s$.

Proof. By Lemma 2.6 we may take a shelling of $\Delta$ in which the facets appear in order of decreasing dimension. In this shelling the facets of dimension less than $r$ 
all appear at the end. This tail may be cut off, which leaves us with a shelling of $\Delta^{(r, d-1)}, d-1=\operatorname{dim} \Delta$.

Suppose that $r<d-1$. It will suffice to prove that $\Delta^{(r, d-2)}$ is shellable. Let

$$
\Delta^{(r, d-1)}=\bigcup_{i=1}^{t}\left[R_{i}, F_{i}\right]
$$

be the Boolean interval partition induced by the shelling. Let $F_{1}, \ldots, F_{k}$ be the facets of dimension $d-1$. For each $i, 1 \leq i \leq k$, choose arbitrarily an order $x_{1}, x_{2}, \ldots, x_{g_{i}}$ of the elements of $F_{i}-R_{i}$, and let $R_{i, j}=R_{i} \cup\left\{x_{1}, \ldots, x_{j-1}\right\}$, $F_{i, j}=F_{i}-\left\{x_{j}\right\}$, for $j=1,2, \ldots, g_{i}$. Then we obtain a disjoint union

$$
\left[R_{i}, F_{i}\right]=\left\{F_{i}\right\} \bigcup_{j=1}^{g_{i}}\left[R_{i, j}, F_{i, j}\right],
$$

and hence a Boolean interval partition

$$
\Delta^{(r, d-2)}=\left(\bigcup_{i=1}^{k} \bigcup_{j=1}^{g_{i}}\left[R_{i, j}, F_{i, j}\right]\right) \dot{\cup}\left(\bigcup_{i=k+1}^{t}\left[R_{i}, F_{i}\right]\right) .
$$

From the fact that property $(\beta)$ of Proposition 2.5 holds for $(*)$ it is easy to deduce that it holds for $(* *)$. Hence, the lexicographic order of the indices induces a shelling order of the facets $F_{i, j}$ and $F_{i}$ of $\Delta^{(r, d-2)}$.

Lemma 2.6 and Theorem 2.9 can be interpreted as providing a kind of "structure theorem", describing how a general shellable complex $\Delta$ is put together from pure shellable complexes. First there is the pure shellable complex $\Delta_{1}=\Delta^{(d-1, d-1)}$ generated by all facets of maximal size. Then $\Delta_{1}$ 's $(d-2)$-skeleton, which is also shellable, is extended by shelling steps in dimension $d-2$ to obtain $\Delta_{2}=\Delta^{(d-2, d-1)}$. Then $\Delta_{2}$ 's $(d-3)$-skeleton is extended by shelling steps in dimension $d-3$ to obtain $\Delta^{(d-3, d-1)}$, and so on until all of $\Delta=\Delta^{(0, d-1)}$ has been constructed.

The general concept of shellability considered here can easily be extended to infinite simplicial complexes. See Section 1 and Remark 4.21 of [B3] for this extension in the pure case.

2.10. Remark. According to Chari $[\mathrm{C}]$ an $\mathcal{S}$-partition of a simplicial complex $\Delta$ is an ordered Boolean interval partition $\Delta=\dot{\bigcup}_{i=1}^{t}\left[R_{i}, S_{i}\right]$ such that $\dot{\bigcup}_{i=1}^{k}\left[R_{i}, S_{i}\right]$ is a complex for all $k$. An $\mathcal{S}$-partition is induced by a shelling as in Proposition 2.5 if and only if all sets $S_{i}$ are facets of $\Delta$. Such $\mathcal{S}$-partitions are called full by Chari; they are mentioned in passing on p. 57 of $[\mathrm{C}]$. Note that every complex has an $\mathcal{S}$-partition, namely the trivial partition into singleton intervals.

One of the main results of $[\mathrm{C}]$ is that certain complexes called "Steiner complexes" of matroids admit nontrivial $\mathcal{S}$-partitions. An example of a Steiner complex is the complex of nonspanning sets of a matroid [C, p. 48], so Example 3.2 below shows that Steiner complexes are not shellable in our sense. 


\section{FACE Numbers AND The $h$-TRIANGLE}

For a $(d-1)$-dimensional complex $\Delta$, let $f_{i}$ be the number of $i$-dimensional faces. The numbers $f_{i}$ are called the face numbers of $\Delta$, and $f(\Delta)=\left(f_{0}, f_{1}, \ldots, f_{d-1}\right)$ its $f$-vector. The $h$-vector $h(\Delta)=\left(h_{0}, h_{1}, \ldots, h_{d}\right)$ is defined by

$$
H(y)=F(y-1),
$$

where $H(y)=\sum_{i=0}^{d} h_{i} y^{d-i}$ and $F(y)=\sum_{i=0}^{d} f_{i-1} y^{d-i}, f_{-1}=1$.

The $h$-vector plays an important role in the theory of Cohen-Macaulay complexes (Stanley [S3, S4, S5]), including pure shellable complexes. To extend part of this to the nonpure case we are led to introduce doubly indexed $f$ - and $h$-numbers.

For $A \in \Delta$, let the size of $A$ be its cardinality and the degree of $A$ be defined by

$$
\delta(A)=\max \{|F| \mid A \subseteq F \in \Delta\} .
$$

Thus, $|A| \leq \delta(A)$, with equality if and only if $A$ is a facet. Also, $\operatorname{dim} l k_{\Delta}(A)=$ $\delta(A)-|A|-1$, where $l k_{\Delta}(A)=\{B \mid B \cap A=\emptyset, B \cup A \in \Delta\}$.

3.1. Definition. For a $(d-1)$-complex $\Delta$, let

(i) $f_{i, j}=$ number of faces of degree $i$ and size $j$,

(ii) $h_{i, j}=\sum_{k=0}^{j}(-1)^{j-k}\left(\begin{array}{c}i-k \\ j-k\end{array}\right) f_{i, k}$,

(iii) the triangular integer arrays $\mathbf{f}=\left(f_{i, j}\right)_{0 \leq j \leq i \leq d}$ and $\mathbf{h}=\left(h_{i, j}\right)_{0 \leq j \leq i \leq d}$ are called the $f$-triangle and $h$-triangle of $\Delta$, respectively.

With the convention that indices $(i, j)$ denote row $i$ and column $j$ we get the following representation of $\mathbf{f}$, and similarly for $\mathbf{h}$ :

$$
\begin{array}{cccc}
f_{0,0} & & & \\
f_{1,0} & f_{1,1} & & \\
\vdots & & \ddots & \\
f_{d, 0} & f_{d, 1} & \cdots & f_{d, d}
\end{array}
$$

Here are a few immediate observations. If $\Delta$ is pure, then only the last row of $\mathbf{f}$ and of $\mathbf{h}$ have nonzero entries, and these last rows are then the ordinary $f$ - and $h$-vectors, up to reindexing. In the general case it is impossible to deduce $\mathbf{f}$ from the ordinary $f$-vector, and similarly for $h$. For instance, there are 1-dimensional complexes with $f$-triangles

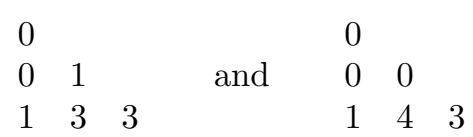

and both have $f$-vector $(4,3)$.

It is clear that

$$
f_{i, j}=\text { number of size } j \text { faces in } \Delta^{(i-1, i-1)} \backslash \Delta^{(i, i)} .
$$


Further, the diagonal entries $\left(f_{i, i}\right)$ of the $f$-triangle give the numbers of facets of various sizes. The column sums give the ordinary face numbers:

$$
f_{j-1}=\sum_{i \geq j} f_{i, j}
$$

Expressing the ordinary $h$-vector in terms of the $h$-triangle is best done using generating functions.

Define the two-variable polynomials

$$
\begin{aligned}
& F(x, y)=\sum_{0 \leq j \leq i} f_{i, j} x^{i} y^{i-j} \\
& H(x, y)=\sum_{0 \leq j \leq i} h_{i, j} x^{i} y^{i-j} .
\end{aligned}
$$

Then relation (ii) of Definition 3.1 can be rewritten as

$$
H(x, y)=F(x, y-1)
$$

and relation (3.3) as

$$
F(y)=y^{d} F\left(\frac{1}{y}, y\right)
$$

From this and equation (3.1) we deduce

$$
H(y)=(y-1)^{d} H\left(\frac{1}{y-1}, y\right),
$$

which is equivalent to

$$
h_{i}=\sum_{j=0}^{i}(-1)^{i-j} \sum_{s=j}^{d-i+j} h_{s, j}\left(\begin{array}{c}
d-s \\
i-j
\end{array}\right),
$$

for $i=0,1, \ldots, d$.

3.2. Example. Let $\Delta$ be the complex whose facets are (omitting commas and set brackets): 123, 345, 14, 15, 24 and 25. Then

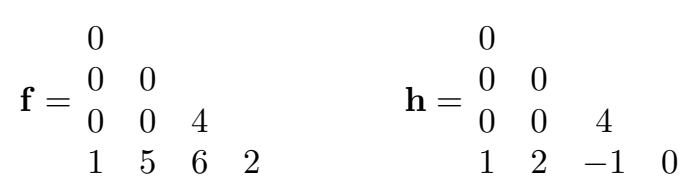

This is the complex of nonspanning sets in the matroid of the graph in Figure 2 (i.e., sets of edges not containing a spanning tree). It is not shellable, since the pure complex $\Delta^{(2,2)}$ generated by 123 and 345 is not shellable; see Theorem 2.9. Being non-shellable is also revealed by the occurrence of a negative entry in the $h$-triangle, as will be shown in Theorem 3.4. 


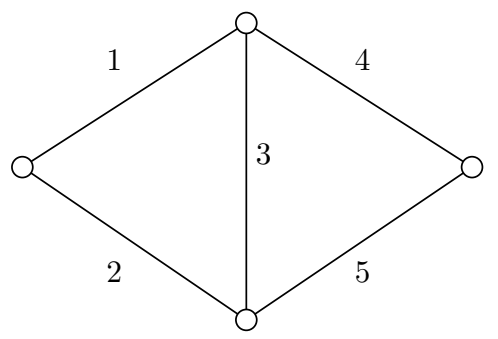

FIGURE 2

As further examples, here are the $h$-triangles of the complexes shown in Figure 1:

$\begin{array}{cccccccc}0 & & & 0 & & & \\ 0 & 1 & & 0 & 0 & & \\ 1 & 0 & 0 & 1 & 2 & -1 & \\ & & & & & & \\ 0 & & & 0 & & & \\ 0 & 0 & & & 0 & 0 & & \\ 0 & 3 & 1 & & 0 & 0 & 1 & \\ 1 & 0 & 0 & 0 & 1 & 3 & -3 & 1\end{array}$

The following lemma gives a few more elementary properties of the $h$-triangle $\left(h_{s, j}\right)_{0 \leq j \leq s \leq d}$ of a $(d-1)$-complex $\Delta$.

3.3. Lemma. (i) $h_{d, 0}=1$ and $h_{s, 0}=0$ for $0 \leq s<d$.

(ii) $\sum_{j=0}^{s} h_{s, j}$ equals the number of $(s-1)$-dimensional facets.

(iii) $\tilde{\chi}(\Delta)=\sum_{j=0}^{d}(-1)^{j-1} h_{j, j}$.

(iv) The h-vector $\left(h_{s, j}^{\prime}\right)_{0 \leq j \leq s \leq c}$ of the $(c-1)$-skeleton $\Delta^{(0, c-1)}$ is given by

$$
\begin{aligned}
& h_{c, j}^{\prime}=h_{c, j}+\sum_{s=c+1}^{d} \sum_{i=0}^{j}\left(\begin{array}{c}
s-c-1+j-i \\
j-i
\end{array}\right) h_{s, i}, \\
& \text { and } h_{s, j}^{\prime}=h_{s, j} \text { for all } 0 \leq j \leq s<c \text {. }
\end{aligned}
$$

(v) The h-vector $\left(h_{s, j}^{\prime \prime}\right)_{0 \leq j \leq s \leq c}$ of $\Delta^{(c-1, c-1)}$ is given by

$$
\begin{array}{lll} 
& h_{c, j}^{\prime \prime}=h_{c, j}^{\prime} & \\
\text { and } & h_{s, j}^{\prime \prime}=0 & \text { for all } 0 \leq j \leq s<c .
\end{array}
$$

Proof. These facts follow from Definition 3.1 by straightforward computation. The formula (iii) for the reduced Euler characteristic can also be obtained by substituting $x=-1$ and $y=0$ in (3.4), or by taking $i=d$ in (3.7) and using that $h_{d}=(-1)^{d-1} \tilde{\chi}(\Delta)$.

The $h$-triangle of a shellable complex is computed by any shelling in the following way. In the pure case this result goes back to McMullen [Mc], who seems to have been first to discover the Boolean interval decomposition (2.2) of a pure shellable complex and its connection with the $h$-vector. 
3.4. Theorem. Let $\Delta$ be a shellable complex with h-triangle $\left(h_{s, j}\right)_{0 \leq j \leq s \leq d}$, and let $\mathcal{R}$ be the restriction map induced by a shelling. Then

$$
h_{s, j}=\text { number of facets } F \text { such that }|F|=s \text { and }|\mathcal{R}(F)|=j \text {. }
$$

Proof. It follows from Lemma 2.6 that

$$
\Delta^{(s-1, s-1)} \backslash \Delta^{(s, s)}=\bigcup_{|F|=s}[\mathcal{R}(F), F]
$$

for all $1 \leq s \leq d$. Because of (3.2) this implies that

$$
\sum_{j=0}^{s} f_{s, j} y^{s-j}=\sum_{j=0}^{s} \tilde{h}_{s, j}(y+1)^{s-j}
$$

where $\tilde{h}_{s, j}$ is defined as the number of facets $F$ of $\Delta$ such that $|F|=s$ and $|\mathcal{R}(F)|=$ $j$. But extracting the coefficient of $x^{s}$ in the identity $F(x, y)=H(x, y+1)$, obtained from (3.4), we get

$$
\sum_{j=0}^{s} f_{s, j} y^{s-j}=\sum_{j=0}^{s} h_{s, j}(y+1)^{s-j} .
$$

Hence, $h_{s, j}=\tilde{h}_{s, j}$.

It follows that the $h$-triangle of a shellable complex has nonnegative entries. More can in fact be said about such $h$-triangles by drawing on known facts about the pure case. For this we must first review some terminology.

For integers $n, k \geq 1$ there exists a unique expansion

$$
n=\left(\begin{array}{c}
a_{k} \\
k
\end{array}\right)+\left(\begin{array}{c}
a_{k-1} \\
k-1
\end{array}\right)+\ldots+\left(\begin{array}{c}
a_{i} \\
i
\end{array}\right)
$$

such that $a_{k}>a_{k-1}>\ldots>a_{i} \geq i \geq 1$. This given, let

$$
\partial^{k}(n)=\left(\begin{array}{c}
a_{k}-1 \\
k-1
\end{array}\right)+\left(\begin{array}{c}
a_{k-1}-1 \\
k-2
\end{array}\right)+\ldots+\left(\begin{array}{c}
a_{i}-1 \\
i-1
\end{array}\right)
$$

Also put $\partial^{k}(0)=0$. A sequence of nonnegative integers $\left(c_{0}, c_{1}, \ldots\right)$ is called an $M$-sequence if $c_{0}=1$ and $\partial^{k}\left(c_{k}\right) \leq c_{k-1}$ for all $k \geq 2$.

3.5. Theorem (Stanley [S3]). A sequence $\left(h_{0}, h_{1}, \ldots, h_{d}\right)$ is the $h$-vector of a pure shellable complex if and only if it is an $M$-sequence.

Using this result we can derive some necessary conditions for the $h$-triangle of arbitrary shellable complexes. 
3.6. Theorem. Let $\left(h_{s, j}\right)_{0 \leq j \leq s \leq d}$ be the $h$-triangle of a shellable complex. Then $h_{s, j} \geq 0$ and the sequence $\left(h_{c, 0}^{\prime}, h_{c, 1}^{\prime}, \ldots, h_{c, c}^{\prime}\right)$ is an $M$-sequence for all $1 \leq c \leq d$, where

$$
h_{c, j}^{\prime}=h_{c, j}+\sum_{s=c+1}^{d} \sum_{i=0}^{j}\left(\begin{array}{c}
s-c-1+j-i \\
j-i
\end{array}\right) h_{s, i} .
$$

Proof. The complex $\Delta^{(c-1, c-1)}$ is pure and shellable by Theorem 2.9. Its $h$-vector is described in part (v) of Lemma 3.3. The result then follows from Theorem 3.5.

Can Stanley's characterization be extended from the pure to the general case? It is conceivable that the necessary conditions of Theorem 3.6 are also sufficient for $h$-triangles of shellable complexes. We leave this question open.

Stanley's Theorem 3.5 gives a characterization of the $f$-vectors of pure shellable complexes, since the $f$-vector and the $h$-vector determine each other. A characterization of the $f$-vectors (but not the $f$-triangles) of $(r, s)$-pure shellable complexes is given in [B7]. Another natural question is to seek a characterization of the triangular arrays that arise as $f$-triangles of simplicial complexes. Since this contains as a special case the characterization of $f$-vectors of pure complexes, this is likely to be a difficult problem.

\section{TOPOLOGICAL PROPERTIES}

It is well known that a pure shellable $(d-1)$-complex has the homotopy type of a wedge of $(d-1)$-spheres. This simplicity of topological structure generalizes, and just as in the pure case a shelling induces bases for homology and cohomology. See e.g. Munkres $[\mathrm{M}]$ for definitions and background concerning the topological notions used. We will not differentiate notationally between a complex $\Delta$ (a set system) and its geometric realization (a topological space).

4.1. Theorem. Let $\Delta$ be a shellable $(d-1)$-complex. Then $\Delta$ has the homotopy type of a wedge of spheres, consisting of $h_{j, j}$ copies of the $(j-1)$-sphere for $1 \leq j \leq d$.

It follows that homology $\widetilde{H}_{*}(\Delta, \mathbb{Z})$ and cohomology $\widetilde{H}^{*}(\Delta, \mathbb{Z})$ are torsion-free, with Betti numbers $\left(h_{j, j}\right)$ that are combinatorially computed by any shelling of $\Delta$, as shown by Theorem 3.4.

4.2. Corollary. $\widetilde{H}_{j-1}(\Delta, \mathbb{Z}) \cong \mathbb{Z}^{h_{j, j}} \cong \widetilde{H}^{j-1}(\Delta, \mathbb{Z})$, for all $j$.

Proof of Theorem 4.1. For some fixed shelling of $\Delta$, let $\Gamma=\{$ facets $F$ such that $\mathcal{R}(F)=F\}$ and $\Delta^{*}=\Delta \backslash \Gamma$. Then by Lemma 2.7 the induced order of the remaining facets is a shelling of $\Delta^{*}$, whose restriction map is $\mathcal{R}$.

Let $F_{k}$ be the $k$-th facet of $\Delta^{*}$ and set $\Delta_{k}^{*}=\bigcup_{i=1}^{k} \bar{F}_{i}$. The facet $F_{k}$ has a free face in $\Delta_{k}^{*}$ (i.e., a face contained in no other facet), namely $\mathcal{R}\left(F_{k}\right)$. This is a proper face, since $\mathcal{R}\left(F_{k}\right) \neq F_{k}$. Thus, removing $\mathcal{R}\left(F_{k}\right)$ and all faces containing it collapses $\Delta_{k}^{*}$ to $\Delta_{k}^{*} \backslash\left[\mathcal{R}\left(F_{k}\right), F_{k}\right]=\Delta_{k-1}^{*}$. It follows that $\Delta_{k-1}^{*}$ and $\Delta_{k}^{*}$ are homotopy equivalent. (In fact, $\Delta_{k-1}^{*}$ is a strong deformation retract of $\Delta_{k}^{*}$.) Since $\Delta_{1}^{*}$ is a simplex, we conclude that $\Delta^{*}$ is contractible.

Now use the fact that passing to the quotient space modulo a contractible subspace does not alter homotopy type: $\Delta \simeq \Delta / \Delta^{*}$ (see [BWa] or [Sp, Coroll. 3.2.5]). The space $\Delta$ is obtained from $\Delta^{*}$ by attaching the cells (simplices) in $\Gamma$, each one 
along its entire boundary. Thus when $\Delta^{*}$ is contracted to a point, each closed $(j-1)$-cell $F \in \Gamma$ is deformed into a $(j-1)$-sphere with a distinguished point $\Delta^{*} / \Delta^{*}$. There are $h_{j, j}=\operatorname{card}\{F \in \Gamma|| F \mid=j\}$ such cells $F$, and the resulting spheres are topologically independent outside the common point $\Delta^{*} / \Delta^{*}$ (the wedge point). Thus we conclude that $\Delta / \Delta^{*}$ is homeomorphic to a wedge of spheres with the required distribution of dimensions.

Let us call the set $\Gamma$ of facets $F$ such that $\mathcal{R}(F)=F$ the homology facets of the shelling. Their crucial property is that the subcomplex $\Delta^{*}=\Delta \backslash \Gamma$ is contractible, and that consequently their enumeration determines the Betti numbers of $\Delta$. They also induce dual bases for homology and cohomology, as we will now see.

Let $C_{k}(\Delta)$ denote the $k$-th simplicial chain group, whose elements are formal linear combinations of $k$-faces of $\Delta$ with integer coefficients. Also, let $C^{k}(\Delta)=$ Hom $\left(C_{k}(\Delta), \mathbb{Z}\right)$ be the $k$-th group of simplicial cochains. The boundary operator $\partial: C_{k}(\Delta) \rightarrow C_{k-1}(\Delta)$ and the coboundary operator $\delta: C^{k-1}(\Delta) \rightarrow C^{k}(\Delta)$ are defined in the standard fashion, as are (co)cycles and (co)boundaries; see Munkres $[\mathrm{M}]$.

Let $\Gamma_{j}=\{F \in \Gamma|| F \mid=j\}$. For each $F \in \Gamma_{j}$ define a $(j-1)$-cochain $\sigma^{F}$ in terms of its values on the basis elements $A \in \Delta_{j-1}=\{(j-1)$-faces of $\Delta\}$ as follows:

$$
\sigma^{F}(A)= \begin{cases}1, & A=F \\ 0, & A \neq F\end{cases}
$$

Since $F$ is a facet of $\Delta$, one sees that $\sigma^{F}$ is a cocycle. Hence it determines a cohomology class $\left[\sigma^{F}\right] \in \widetilde{H}^{j-1}(\Delta, \mathbb{Z})$.

4.3. Theorem. Let $\Gamma_{j}$ be the set of homology facets of size $j$ induced by a shelling of $\Delta$. Then the classes $\left[\sigma^{F}\right]$, for $F \in \Gamma_{j}$, are a basis of $\widetilde{H}^{j-1}(\Delta, \mathbb{Z})$.

Proof. Let $\rho$ be any cocycle in $C^{j-1}(\Delta)$. Consider the cocycle

$$
\tau=\rho-\sum_{F \in \Gamma_{j}} \rho(F) \sigma^{F}
$$

We have that $\tau(F)=0$ for all $F \in \Gamma_{j}$, so $\tau$ is in fact a cocycle in $C^{j-1}\left(\Delta^{*}\right)$. But $\widetilde{H}^{j-1}\left(\Delta^{*}, \mathbb{Z}\right)=0$, since $\Delta^{*}$ is contractible. Hence $\tau=\delta_{\Delta^{*}}\left(\tau^{\prime}\right)$ for some $\tau^{\prime} \in C^{j-2}\left(\Delta^{*}\right)$. This implies that $\delta_{\Delta}\left(\tau^{\prime}\right)=\tau+\sum_{F \in \Gamma_{j}} a_{F} \sigma^{F}$, and hence

$$
[\rho]=\sum_{F \in \Gamma_{j}}\left(\rho(F)-a_{F}\right)\left[\sigma^{F}\right]
$$

We know from Corollary 4.2 that $\widetilde{H}^{j-1}(\Delta, \mathbb{Z})$ is a free Abelian group of rank $h_{j, j}$, and it has just been shown that it is generated by the $\left|\Gamma_{j}\right|=h_{j, j}$ elements $\left[\sigma^{F}\right]$. Hence, these elements form a basis.

Since the homology of a shellable complex is free, the isomorphism between homology and cohomology (Corollary 4.2) takes the form of a certain duality pairing $\langle\sigma, \tau\rangle$ (see Munkres [M, pp. 276-277]). Hence, corresponding to the cohomology classes $\left[\sigma^{F}\right]$ there are dual homology classes $\left[\sigma_{F}\right], F \in \Gamma_{j}$, determined by $\left\langle\sigma^{G}, \sigma_{F}\right\rangle=\delta_{G, F}$. In concrete terms this means that $(j-1)$-cycles $\sigma_{F}, F \in \Gamma_{j}$, are uniquely determined (modulo boundaries) by the property that $\sigma_{F}$ has coefficient +1 at the $(j-1)$-face $F$ and coefficient 0 at all other homology facets $\Gamma_{j}$. The following is a consequence of (and in fact equivalent to) Theorem 4.3. 
4.4. Corollary. The classes $\left[\sigma_{F}\right], F \in \Gamma_{j}$, are a basis of $\widetilde{H}_{j-1}(\Delta, \mathbb{Z})$.

For a direct and elementary proof of Corollary 4.4 in the case of pure shellable complexes, see Theorem 7.7.2 of [B4]. The details of that proof can be adapted to the nonpure case as well.

The only property of a shellable complex $\Delta$ that has been used in this section is the existence of a set $\Gamma$ of facets whose removal leaves a contractible subcomplex $\Delta^{*}=\Delta \backslash \Gamma$. Hence, all results are valid for the larger class of all complexes with this property.

\section{LEXICOGRAPHICALLY SHELlABLE POSETS}

With any finite poset $P$ we associate the order complex $\Delta(P)$ whose $k$-faces are the chains (totally ordered subsets) $x_{0}<x_{1}<\ldots<x_{k}$ of $P$. Properties of complexes, such as purity, shellability and topological type, can be transferred to posets via order complexes.

A special method for the study of $\Delta(P)$ for pure posets via certain labelings of the cover relations (R-labelings, EL-labelings, CL-labelings) have been introduced and developed in a series of papers by the authors [B1, BW1, BW2]. The resulting theory of "lexicographic shellability" will here be generalized to the case of nonpure posets.

A poset is called bounded if it has a top element $\hat{1}$ and a bottom element $\hat{0}$. If $P$ is bounded, let $\bar{P}=P \backslash\{\hat{0}, \hat{1}\}$. Conversely, for any poset $P$ let $\hat{P}=P \cup\{\hat{0}, \hat{1}\}$, where $\hat{0}$ and $\hat{1}$ are new elements adjoined so that $\hat{0}<x<\hat{1}$ for all $x \in P$. If $x \leq y$, we have the closed interval $[x, y]=\{z \in P \mid x \leq z \leq y\}$ and the open interval $(x, y)=\{z \in P \mid x<z<y\}$. We say that $y$ covers $x$, and write $x \rightarrow y$, if $x<y$ and $(x, y)=\emptyset$. Let $\mathcal{E}(P)=\{(x, y) \in P \times P \mid x \rightarrow y\}$ be the covering relation, which is the same thing as the edges of the Hasse diagram of $P$. The length of a chain $c: x_{0}<x_{1}<\ldots<x_{k}$ is $k$, written $\ell(c)=k$, and the length of $P$, denoted $\ell(P)$, is the length of the longest chain in $P$.

A chain in $P$ is maximal if it is inclusionwise maximal. Thus, the set $\mathcal{M}(P)$ of maximal chains is the set of facets of $\Delta(P)$. A chain $x_{0}<x_{1}<\ldots<x_{k}$ is unrefinable if it is maximal in the interval $\left[x_{0}, x_{k}\right]$.

For a bounded poset $P$ let $\mathcal{M E}(P)$ be the set of pairs $(m, x \rightarrow y) \in \mathcal{M}(P) \times \mathcal{E}(P)$ consisting of a maximal chain $m$ and an edge $x \rightarrow y$ along that chain (i.e., $x, y \in m$ ).

5.1. Definition. (i) An edge labeling of $P$ is a map $\lambda: \mathcal{E}(P) \rightarrow \Lambda$, where $\Lambda$ is some poset.

(ii) A chain-edge labeling of $P$ is a map $\lambda: \mathcal{M E}(P) \rightarrow \Lambda$, where $\Lambda$ is some poset, satisfying:

Axiom CE: If two maximal chains $m: \hat{0}=x_{0} \rightarrow x_{1} \rightarrow \ldots \rightarrow x_{k}=\hat{1}$ and $m^{\prime}: \hat{0}=x_{0}^{\prime} \rightarrow x_{1}^{\prime} \rightarrow \ldots \rightarrow x_{n}^{\prime}=\hat{1}$ coincide along their first $d$ edges, then their labels also coincide along these edges. That is, if $x_{i}=x_{i}^{\prime}$ for $i=0,1, \ldots, d$, then $\lambda\left(m, x_{i-1} \rightarrow x_{i}\right)=\lambda\left(m^{\prime}, x_{i-1}^{\prime} \rightarrow x_{i}^{\prime}\right)$ for $i=1, \ldots, d$.

An edge labeling $\lambda$ naturally induces a chain-edge labeling $\lambda^{\prime}$ by letting $\lambda^{\prime}(m, x \rightarrow y)=\lambda(x \rightarrow y)$ for all maximal chains $m$ containing the edge $x \rightarrow y$. Examples of both kinds of labelings for the pure case can be found in [BW2]. Two examples of edge labelings of nonpure posets are given in Figure 3. 


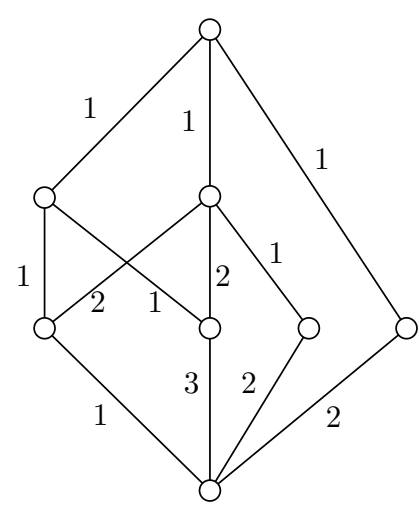

(a)

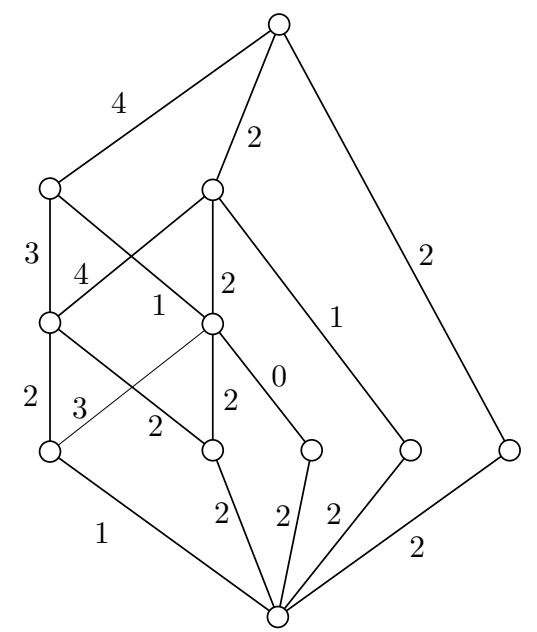

(b)

FiguRE 3

Let $\lambda$ be a chain-edge labeling of a bounded poset $P$. With each maximal chain $m: \hat{0}=x_{0} \rightarrow x_{1} \rightarrow \ldots \rightarrow x_{n}=\hat{1}$ we associate the ordered string

$$
\lambda(m)=\left[\lambda\left(m, x_{0} \rightarrow x_{1}\right), \ldots, \lambda\left(m, x_{n-1} \rightarrow x_{n}\right)\right] .
$$

Note that the length of the tuple $\lambda(m)$ depends on the length of the chain $m$.

If $[x, y]$ is an interval and $r$ is an unrefinable chain from $\hat{0}$ to $x$, then the pair $([x, y], r)$ will be called a rooted interval with root $r$, and will be denoted $[x, y]_{r}$. If $m$ is any maximal chain of $[x, y]$, we shall also consider it a maximal chain of the rooted interval $[x, y]_{r}$. Then $r \cup m$ is a maximal chain of $[\hat{0}, y]$.

Let $\lambda$ be a chain-edge labeling of $P$ and $[x, y]_{r}$ a rooted interval. By axiom CE, if $m$ is a maximal chain of $[x, y]_{r}$ and $m^{\prime}$ and $m^{\prime \prime}$ are maximal chains of $P$ that contain $r \cup m$, then the first $d$ entries of $\lambda\left(m^{\prime}\right)$ and $\lambda\left(m^{\prime \prime}\right)$ coincide, where $d=\ell(r \cup m)$. Hence, if we remove the first $\ell(r)$ entries from this $d$-tuple (the part belonging to $r)$, we get a $(d-\ell(r))$-tuple $\lambda_{r}(m)$ associated with $m$, and uniquely induced by any maximal chain in $P$ that contains $r \cup m$. See Figure 4 .

The labels $\lambda_{r}(m)$ that we are working with are ordered strings of elements from the poset $\Lambda$. Their lengths may vary. Let $\Lambda^{*}$ denote the set of all such strings (or "words in the partially ordered alphabet $\Lambda$ "). We will use the lexicographic partial order on $\Lambda^{*}$, defined by

$$
\left(a_{1}, \ldots, a_{p}\right) \leq_{L}\left(b_{1}, \ldots, b_{q}\right)
$$

if and only if either

(i) $a_{i}=b_{i}$ for $i=1, \ldots, p$ and $p \leq q$, or,

(ii) $a_{i} \neq b_{i}$ for some $i$ and $a_{i}<b_{i}$ for the least such $i$.

5.2. Definition. Let $\lambda: \mathcal{M E}(P) \rightarrow \Lambda$ be a chain-edge labeling of a bounded poset $P$.

1. $\lambda$ is called a CR-labeling (chain rising labeling) if in every rooted interval $[x, y]_{r}$ 


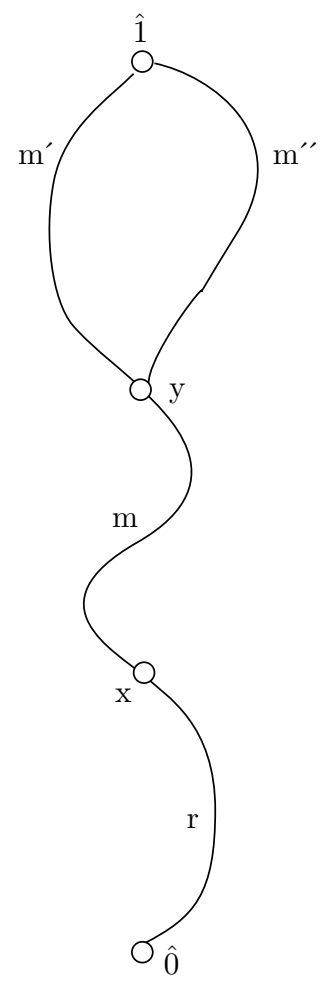

FIGURE 4

of $P$ there is a unique maximal chain $m$ whose label $\lambda_{r}(m)=\left(a_{1}, \ldots, a_{p}\right)$ satisfies $a_{1}<a_{2}<\ldots<a_{p}$ in $\Lambda$. We call $m$ the rising chain in $[x, y]_{r}$.

2. A CR-labeling $\lambda$ is called a $C L$-labeling (chain lexicographic labeling) if for every $[x, y]_{r}$ the unique rising chain $m$ is lexicographically strictly first, that is, $\lambda_{r}(m)<\lambda_{r}\left(m^{\prime}\right)$ for all other maximal chains $m^{\prime}$ in $[x, y]_{r}$.

3. A CR- or CL-labeling that comes from an edge labeling $\lambda: \mathcal{E}(P) \rightarrow \Lambda$ will be called an ER- or EL-labeling, respectively. (For edge rising/lexicographic labeling.)

4. A bounded poset that admits an $E L$ - or $C L$-labeling will be called $E L$ - or $C L$-shellable (edge/chain lexicographically shellable).

Some variation is possible in the concept of "rising chain" used in this definition. Most importantly, one could require nonstrict inequalities instead: $a_{1} \leq a_{2} \leq \ldots \leq$ $a_{p}$. See Remark 5.14 for more about this. Figure 3 (a) shows an "alternative" ELlabeling whose rising chains are weakly increasing, while (b) shows a "standard" EL-labeling with strictly increasing rising chains.

Let us say a few words about the history of these definitions in the pure case. Stanley defined the concept of an admissible lattice $[\mathrm{S} 1, \mathrm{~S} 2]$ as a pure lattice $L$ with a map $\omega: I(L) \rightarrow \mathbb{Z}$ from the join-irreducibles $I(L)$ such that the induced edge labeling

$$
\lambda(x \rightarrow y)=\min \{\omega(z) \mid z \in I(L), x<x \vee z=y\}
$$


has exactly one increasingly labeled chain in every interval. The motivation was to combinatorially interpret and prove nonnegativity of the rank-selected Möbius invariants $(-1)^{|J|+1} \mu\left(L_{J}\right)$. The two main examples of admissible lattices were semimodular and supersolvable lattices [S2]. Stanley also conjectured in [S3] that admissible lattices are Cohen-Macaulay. This conjecture was proved by Björner [B1] using shellability. In [B1] the step was taken to consider labelings of edges of a poset (rather than labeling of join-irreducibles of a lattice) as the primitive operation, and the concepts of $R$-labeling (for Möbius function results à la Stanley) and $L$-labeling (for shellability and Cohen-Macaulayness) were introduced. This is the pure case of what is here called ER- and EL-labeling. The step to the greater generality of chain-edge labelings was taken by the authors in [BW1] to prove the shellability (and hence Cohen-Macaulayness) of Bruhat order of Coxeter groups. A more detailed treatment of pure CL-labelings followed in [BW2].

CL-labelings can be recognized among CR-labelings by the following criterion.

5.3. Lemma. A CR-labeling $\lambda$ is a CL-labeling if and only if the following holds for every rooted interval $[x, y]_{r}$ : if $a_{1}, a_{2}, \ldots, a_{k}$ are the atoms of $[x, y]$ and $a_{1}$ lies on the unique rising chain of $[x, y]_{r}$, then $\lambda\left(x \rightarrow a_{1}\right)<\lambda\left(x \rightarrow a_{i}\right), i=2, \ldots, k$. Here $\lambda\left(x \rightarrow a_{i}\right)$ are the edge-labels induced by the chain-edge labeling $\lambda$ and the root $r$.

Proof. See the proof of Proposition 2.5 in [B1].

Let $\lambda: \mathcal{M E}(P) \rightarrow \Lambda$ be a CR-labeling of a bounded poset $P$. We need the following concepts.

5.4. Definition. For a maximal chain $m: \hat{0}=x_{0} \rightarrow x_{1} \rightarrow \ldots \rightarrow x_{k+1}=\hat{1}$,

(a) $\mathcal{D}(m)=\left\{i \mid \lambda\left(m, x_{i-1} \rightarrow x_{i}\right) \nless \lambda\left(m, x_{i} \rightarrow x_{i+1}\right)\right\}$ is called the descent set of $m$,

(b) $\mathcal{R}(m)=\left\{x_{i} \in m \mid i \in \mathcal{D}(m)\right\}$ is the restriction of $m$,

(c) $m$ is said to be falling if $\mathcal{D}(m)=\{1,2, \ldots, k\}$.

Let $\Delta(\bar{P})$ be the order complex of the proper part $\bar{P}=P \backslash\{\hat{0}, \hat{1}\}$ of our bounded poset. Let $\bar{m}=m \backslash\{\hat{0}, \hat{1}\}$ for maximal chains $m$ in $P$. Note that $\mathcal{R}(m) \subseteq \bar{m}$. We get a Boolean interval partition as follows.

5.5. Proposition. A CR-labeling of $P$ induces a partition

$$
\Delta(\bar{P})=\bigcup_{m \in \mathcal{M}}^{\cdot}[\mathcal{R}(m), \bar{m}] .
$$

Proof. Let $c: y_{1}<y_{2}<\ldots<y_{e}$ be a chain in $\bar{P}$. Construct a maximal chain containing $c$ as follows. Let $m_{1}$ be the unique rising chain in $\left[\hat{0}, y_{1}\right]$. Then let $m_{2}$ be the rising chain in the rooted interval $\left[y_{1}, y_{2}\right]_{m_{1}}$. We continue by letting $m_{i+1}$ be the rising chain in $\left[y_{i}, y_{i+1}\right]_{m_{1} \cup \ldots \cup m_{i}}$, until we obtain a maximal chain $m=m_{1} \cup \ldots \cup m_{e+1}$. Then by construction $\mathcal{R}(m) \subseteq c \subseteq \bar{m}$.

If $\mathcal{R}\left(m^{\prime}\right) \subseteq c \subseteq \bar{m}^{\prime}$ for some maximal chain $m^{\prime}$, then there is no descent along $m^{\prime} \cap\left[y_{i-1}, y_{i}\right], i=1,2, \ldots$ Hence, $m^{\prime} \cap\left[y_{i-1}, y_{i}\right]$ is the unique rising chain in the rooted interval $\left[y_{i-1}, y_{i}\right]_{m^{\prime} \cap\left[\hat{o}, y_{i-1}\right]}$. This determines $m^{\prime}$ uniquely.

Before going further, let us formally state the simple fact that CR/CL-labelings are hereditary on intervals. Therefore, all facts we will prove about posets $P$ with such a labeling are automatically true for all intervals $[x, y]$ in $P$. 
5.6. Lemma. Let $\lambda: \mathcal{M E}(P) \rightarrow \Lambda$ be a CR-or CL-labeling of $P$ and let $[x, y]_{r}$ be a rooted interval. Then $\lambda_{r}: \mathcal{M E}([x, y]) \rightarrow \Lambda$ is a CR-resp. CL-labeling of the interval $[x, y]$.

Proof. Directly from Definition 5.2.

In agreement with this and Definition 5.4 a chain $m$ in a rooted interval $[x, y]_{r}$ is called falling if $m$ is maximal in $[x, y]$ and $\lambda_{r}(m)=\left(a_{1}, \ldots, a_{p}\right)$ with $a_{1} \nless a_{2} \nless$ $\ldots \nless a_{p}$.

The Möbius function of a bounded poset can be computed from a CR-labeling in the following way. Here the root $r$ can be chosen arbitrarily. If we have an ER-labeling, or if $x=\hat{0}$, the dependence on $r$ disappears.

5.7. Proposition. $\mu(x, y)=$ number of even length falling chains in $[x, y]_{r}$ - number of odd length falling chains in $[x, y]_{r}$.

Proof. Lemma 5.6 shows that it suffices to consider $x=\hat{0}, y=\hat{1}$. By the theorem of Ph. Hall [S5, p. 120] $\mu_{P}(\hat{0}, \hat{1})$ equals the number of chains of even length minus the number of chains of odd length in $\bar{P}$. The contribution to this sum from each interval $[\mathcal{R}(m), \bar{m}]$ in Proposition 5.5 is $=0$ if $\mathcal{R}(m) \neq \bar{m}$, and $=(-1)^{\ell(m)}$ if $\mathcal{R}(m)=\bar{m}$, i.e. if $m$ is falling.

For example, the poset $P_{1}$ in Figure 3 (a) has 2 falling maximal chains, of lengths 2 and 3 , so $\mu_{P_{1}}(\hat{0}, \hat{1})=0$. The poset $P_{2}$ in Figure 3 (b) also has 2 falling chains, but now of lengths 2 and 4 , so $\mu_{P_{2}}(\hat{0}, \hat{1})=2$.

5.8. Theorem. If a bounded poset $P$ is $C L$-shellable, then $\Delta(\bar{P})$ is shellable. More precisely, given a CL-labeling of $P$ any ordering of the maximal chains of $P$ that extends the lexicographic partial order of their labels is a shelling, whose restriction map coincides with the map $\mathcal{R}$ of Definition 5.4.

Proof. In view of Propositions 2.5 and 5.5 it remains to be shown that if $\mathcal{R}(m) \subseteq m^{\prime}$, for $m \neq m^{\prime} \in \mathcal{M}(P)$, then $\lambda(m)<\lambda\left(m^{\prime}\right)$ in lexicographic order.

Let $m: \hat{0}=x_{0} \rightarrow x_{1} \rightarrow \ldots$ and $m^{\prime}: \hat{0}=x_{0}^{\prime} \rightarrow x_{1}^{\prime} \rightarrow \ldots$, and let $i$ be minimal such that $x_{i+1} \neq x_{i+1}^{\prime}$. Then let $y$ be minimal such that $y \in m \cap m^{\prime}$ and $x_{i}=x_{i}^{\prime}<y$. Since $\mathcal{R}(m) \subseteq m \cap m^{\prime}$, it follows that there is no descent along $m \cap\left[x_{i}, y\right]$. Hence, $m \cap\left[x_{i}, y\right]$ is the rising chain in the rooted interval $\left[x_{i}, y\right]_{m \cap\left[\hat{0}, x_{i}\right]}$, and it follows from Lemma 5.3 that $\lambda\left(m, x_{i} \rightarrow x_{i+1}\right)<\lambda\left(m^{\prime}, x_{i}^{\prime} \rightarrow x_{i+1}^{\prime}\right)$.

Theorem 5.8 can also be proven directly from the definition of shellability. For this argument in the pure case (which generalizes straightforwardly), see [BW1, Theorem 3.3]. A property of $\Delta(\bar{P})$ that is stronger than shellability will be proven in Theorem 11.6.

It follows that the homology facets of a CL-shellable poset (for some fixed CLlabeling) are the falling chains. Hence we can summarize the following conclusions from the results of Section 4.

5.9. Theorem. If a bounded poset $P$ is $C L$-shellable, then $\Delta(\bar{P})$ has the homotopy type of a wedge of spheres. Furthermore, for any fixed CL-labeling:

(i) $\widetilde{H}_{i}(\Delta(\bar{P}), \mathbb{Z}) \cong \mathbb{Z}^{\# \text { falling chains of length } i+2}$,

(ii) bases for $i$-dimensional homology and cohomology are induced by the falling chains of length $i+2$, as explained in connection with Theorem 4.3. 
For instance, we obtain the nonzero Betti numbers $\tilde{\beta}_{0}=\tilde{\beta}_{1}=1$ for the poset in Figure $3(\mathrm{a})$, and $\tilde{\beta}_{0}=\tilde{\beta}_{2}=1$ for the one in Figure $3(\mathrm{~b})$.

An alternative approach to lexicographic shellability, via so-called "recursive atom orderings" was introduced in [BW2]. This extends without difficulty to nonpure posets. Recall that the atoms of a bounded poset are the elements that cover $\hat{0}$.

5.10. Definition. A bounded poset $P$ is said to admit a recursive atom ordering if the length of $P$ is 1 , or if $\ell(P)>1$ and there is an ordering $a_{1}, a_{2}, \ldots, a_{t}$ of the atoms of $P$ that satisfies:

(i) For all $j=1,2, \ldots, t$ the interval $\left[a_{j}, \hat{1}\right]$ admits a recursive atom ordering in which the atoms of $\left[a_{j}, \hat{1}\right]$ that belong to $\left[a_{i}, \hat{1}\right]$ for some $i<j$ come first.

(ii) For all $i<j$, if $a_{i}, a_{j}<y$, then there is a $k<j$ and an atom $z$ of $\left[a_{j}, \hat{1}\right]$ such that $a_{k}<z \leq y$.

A recursive coatom ordering is a recursive atom ordering of the dual poset $\left(P^{*}, \leq\right)=$ $(P, \geq)$.

The proof of Theorem 3.2 of [BW2] goes through with only notational adjustments in the nonpure case, and we obtain the following.

5.11. Theorem. A bounded poset $P$ admits a recursive atom ordering if and only if $P$ is $C L$-shellable.

The proof of sufficiency produces an integer CL-labeling. Hence the following conclusion can be drawn, just as in the pure case.

5.12. Corollary. If $P$ admits a $C L$-labeling $\mathcal{M E} \rightarrow \Lambda$, for any poset $\Lambda$, then $P$ admits a $C L$-labeling $\mathcal{M E} \rightarrow(\mathbb{Z},<)$.

Let $\hat{L}(\Delta)$ be the face lattice of a complex $\Delta$, i.e., the set of all faces ordered by inclusion and with a top element $\hat{1}$ adjoined. Say that a poset $P$ is dual $C L$-shellable if its dual poset $P^{*}$ obtained by reversing the order relation is CL-shellable. The following generalizes Theorem 4.3 of [BW2].

5.13. Theorem. For any complex $\Delta$ the following are equivalent:

(i) $\Delta$ is shellable,

(ii) $\hat{L}(\Delta)$ admits a recursive coatom ordering,

(iii) $\hat{L}(\Delta)$ is dual CL-shellable.

Proof. The equivalence (i) $\Longleftrightarrow$ (ii) has the same proof as in the pure case; see Theorem 4.3 of [BW2]. The equivalence (ii) $\Longleftrightarrow$ (iii) is a special case of Theorem 5.11.

5.14. Remark. In Definition 5.2 we have required that the label $\lambda_{r}(m)=\left(a_{1}, \ldots, a_{p}\right)$ of a rising chain is strictly increasing: $a_{1}<a_{2}<\ldots<a_{p}$. We could instead require that it is weakly increasing: $a_{1} \leq a_{2} \leq \ldots \leq a_{p}$. The results of this section still hold, except that the notion of descent set $\mathcal{D}(m)$ of a maximal chain $m$ (Definition 5.4) must be altered to mean $\mathcal{D}(m)=\left\{i \mid \lambda\left(m, x_{i-1} \rightarrow x_{i}\right) \not \leq \lambda\left(m, x_{i} \rightarrow x_{i+1}\right)\right\}$. The definition of restriction $\mathcal{R}(m)$ and of falling chain must be altered accordingly.

To reduce the "weakly increasing" version to the "strictly increasing" version, used in Definition 5.2, just take as new poset of labels the direct product $\Lambda^{\prime}=\Lambda \times \mathbb{Z}$ and relabel $m: \hat{0}=x_{0} \rightarrow x_{1} \rightarrow \ldots \rightarrow x_{k}=\hat{1}$ by

$$
\lambda^{\prime}\left(m, x_{i-1} \rightarrow x_{i}\right)=\left(\lambda\left(m, x_{i-1} \rightarrow x_{i}\right), i\right) .
$$


Note that this relabeling will convert a "weakly increasing" EL-labeling to a "strictly increasing" CL-labeling in the nonpure case.

To convert a "strictly increasing" CL-labeling to a "weakly increasing" CLlabeling, take as the label poset lexicographical order on $\Lambda \times \mathbb{Z}$ and relabel $m$ : $\hat{0}=x_{0} \rightarrow x_{1} \rightarrow \ldots \rightarrow x_{k}=\hat{1}$ by

$$
\lambda^{\prime}\left(m, x_{i-1} \rightarrow x_{i}\right)=\left(\lambda\left(m, x_{i-1} \rightarrow x_{i}\right),-i\right) .
$$

Note that $\mathcal{D}(m)$ and $\mathcal{R}(m)$ are unchanged by these conversions. This correspondence between the "strictly increasing" version and the "weakly increasing" version in the pure case is discussed in [W].

The possibility to generalize the concept of "rising chain" is actually much wider. Instead of a poset, we can take as label set $\Lambda$ the vertices of an acyclic directed graph, with loops allowed. Write $a \Rightarrow b$ for edges of the graph $\Lambda$. Then a chainedge labeling $\lambda: \mathcal{M E}(P) \rightarrow \Lambda$ is a CR-labeling if in every rooted interval $[x, y]_{r}$ there is a unique maximal chain $m$ whose label $\lambda_{r}(m)=\left(a_{1}, a_{2}, \ldots, a_{p}\right)$ is a path, i.e. satisfies $a_{1} \Rightarrow a_{2} \Rightarrow \ldots \Rightarrow a_{p}$. It is a $C$ L-labeling if in addition if $m^{\prime}$ is any other maximal chain in $[x, y]_{r}$ and $\lambda_{r}\left(m^{\prime}\right)=\left(b_{1}, b_{2}, \ldots, b_{q}\right)$, then $a_{j} \neq b_{j}$ for some $j \leq \min (p, q)$ and $a_{j} \Rightarrow b_{j}$ for the least such $j$. All results of this section, suitably modified notationally, go through in this generality. The acyclic graph used in Definition 5.2 is the comparability graph of a poset $\Lambda$, and for the "weakly increasing" version discussed above we have just added loops at the vertices of that comparability graph.

\section{The $k$-EQUal PARTITION LATTICE}

Let $\Pi_{n}$ be the lattice of partitions of the set $[n]=\{1,2, \ldots, n\}$ ordered by refinement, and for $2 \leq k \leq n$ let

$$
\Pi_{n, k}=\left\{\pi \in \Pi_{n} \mid \text { no block of } \pi \text { has size } 2,3, \ldots, \text { or } k-1\right\} .
$$

Then $\Pi_{n, k}$ is a lattice in the induced order, called the $k$-equal partition lattice. It is isomorphic to the intersection lattice of the arrangement of subspaces given by $x_{i_{1}}=x_{i_{2}}=\ldots=x_{i_{k}}, 1 \leq i_{1}<\ldots<i_{k} \leq n$, in $\mathbb{R}^{n}$.

The lattices $\Pi_{n, k}$ were first studied in connection with a problem in complexity theory in [BLY, BL]. Then their homotopy type and Betti numbers were determined in [BWe]. In this section we will show that nonpure lexicographic shellability gives an effective approach to the analysis of $\Pi_{n, k}$.

We will now describe an edge-labeling $\lambda: \mathcal{E}\left(\Pi_{n, k}\right) \rightarrow \Lambda$, where $\Lambda$ is the linearly ordered set

$$
\overline{1}<\overline{2}<\ldots<\bar{n}<1<2<\ldots<n .
$$

There are three kinds of coverings in $\Pi_{n, k}$, described in the following labeling rule:

$$
\text { Covering } \pi \rightarrow \sigma
$$

A new $k$-block $B$ is created from singletons A nonsingleton block $B$ is merged with a singleton $\{a\}$ Two nonsingleton blocks $B_{1}$ and $B_{2}$ are merged

$$
\begin{aligned}
& \text { Label } \lambda(\pi \rightarrow \sigma) \\
& \lambda=\max B \\
& \lambda=a \\
& \lambda=\overline{\max \left(B_{1} \cup B_{2}\right)}
\end{aligned}
$$




\subsection{Theorem. The labeling $\lambda$ of $\Pi_{n, k}$ is an EL-labeling.}

Proof. Consider first an upper interval $[\pi, \hat{1}]$. Suppose that $\pi$ has nonsingleton blocks $B_{1}, B_{2}, \ldots, B_{p}, p \geq 1$, listed so that their maximal elements $b_{i}=\max B_{i}$ satisfy $b_{1}<b_{2}<\ldots<b_{p}$. Let $\left\{a_{1}\right\},\left\{a_{2}\right\}, \ldots,\left\{a_{q}\right\}$ be the singleton blocks of $\pi$, $a_{1}<a_{2}<\ldots<a_{q}$.

Construct a maximal chain $m$ of length $p+q-1$ in $[\pi, \hat{1}]$ as follows. First merge $B_{1} \cup B_{2}$. Then add successively $B_{3}, B_{4}, \ldots, B_{p}$ to this special block, followed by adding $a_{1}, a_{2}, \ldots$, and finally $a_{q}$. This creates a chain $m$ with rising label $\lambda(m)=$ $\left(\bar{b}_{2}, \bar{b}_{3}, \ldots, \bar{b}_{p}, a_{1}, a_{2}, \ldots, a_{q}\right)$. It is easy to see that $\lambda(m)$ is lexicographically first among the labels of all maximal chains of $[\pi, \hat{1}]$, and also that no other such label is rising.

If $\pi$ has no nonsingleton blocks, i.e. if $\pi=\hat{0}$, then the canonical rising chain is constructed by first creating the $k$-block $\{1,2, \ldots, k\}$ and then successively adding the singletons $k+1, k+2, \ldots$ to this special block. This chain has label $(k, k+$ $1, \ldots, n)$, is lexicographically first and unique with rising label.

Consider now a general interval $[\pi, \sigma]$ in $\Pi_{n, k}$. Suppose that $\sigma$ has nonsingleton blocks $C_{1}, C_{2}, \ldots, C_{r}$. Let $\pi_{i}$ be the partition obtained from $\pi$ by merging all blocks contained in $C_{i}$ and leaving all other blocks unchanged, for $i=1, \ldots, r$. Then each interval $\left[\pi, \pi_{i}\right]$ is isomorphic to an upper interval in the $k$-equal partition lattice on the set $C_{i}$, and with the preceding construction we can create a maximal chain $m_{i}$ in $\left[\pi, \pi_{i}\right]$ whose label $\lambda\left(m_{i}\right)$ is unique rising and lexicographically first. Note that the labels $\lambda\left(m_{i}\right)$ and $\lambda\left(m_{j}\right)$ are disjoint if $i \neq j$, since the entries in $\lambda\left(m_{i}\right)$ are elements of $C_{i}$.

Let $\left\{x_{1}, x_{2}, \ldots, x_{s}\right\}$ be the total set of all entries appearing in any label $\lambda\left(m_{i}\right)$, $i=1, \ldots, r$, and suppose that $x_{1}<x_{2}<\ldots<x_{s}$. We can create a maximal chain $m$ in $[\pi, \sigma]$ with label $\lambda(m)=\left(x_{1}, x_{2}, \ldots, x_{s}\right)$ by building the chains $m_{i}$ in parallel. The rule for the $j$-th step of $m$ is to find $i$ such that $x_{j} \in \lambda\left(m_{i}\right)$, and then to perform the merging of blocks that created a covering with label $x_{j}$ in $m_{i}$. This chain $m$ is the canonical rising chain - we leave to the reader the easy verification that it is unique rising and lexicographically first.

As a first consequence of lexicographic shellability we can determine the topological properties of $\Pi_{n, k}$.

6.2. Corollary (Björner and Welker [BWe]). The order complex $\Delta_{n, k}=\Delta\left(\overline{\Pi_{n, k}}\right)$ has the homotopy type of a wedge of spheres. Let $\tilde{\beta}_{n, k}^{d}$ be the rank of the free Abelian group $\widetilde{H}_{d}\left(\Delta_{n, k}\right)$. Then $\tilde{\beta}_{n, k}^{d} \neq 0$ if and only if $d=n-3-t(k-2)$ for some $t$, $1 \leq t \leq\left\lfloor\frac{n}{k}\right\rfloor$, and

$$
\tilde{\beta}_{n, k}^{n-3-t(k-2)}=(t-1) ! \sum_{0=i_{0} \leq \ldots \leq i_{t}=n-t k} \prod_{j=0}^{t-1}\left(\begin{array}{c}
n-j k-i_{j}-1 \\
k-1
\end{array}\right)(j+1)^{i_{j+1}-i_{j}} .
$$

Proof. The general result follows from Theorem 5.9, which also shows that $\tilde{\beta}_{n, k}^{d}$ equals the number of falling chains of length $d+2$.

Let the maximal chain $m$ be falling, that is, its label $\lambda(m)$ satisfies $\lambda_{1} \geq \lambda_{2} \geq \ldots$. Then all unbarred labels must precede all barred ones. This means that in the formation of $m$ there is a first stage consisting of creations of $k$-blocks and mergings 
with singletons, and a second stage consisting entirely of mergings of non-singleton blocks. Suppose that $t k$-blocks are created during the first stage. Then $t$ is also the number of non-singleton blocks at the beginning of the second stage. The creation of a $k$-block reduces the number of blocks in the current partition by $k-1$, and all other steps reduce this number by one. Hence the length of $m$ is $n-1-t(k-2)$.

Let us now count the number of falling chains of length $n-1-t(k-2)$. The first stage in the formation of such a chain consists in the creation of $t k$-blocks interspersed with mergings with singletons. It begins with creating the first $k$ block, which must contain $n$, and after the $t$-th $k$-block is created, the remaining singletons (if any) are merged until none remain.

Let $0=i_{0} \leq \ldots \leq i_{t}=n-t k$. We claim that

$$
\prod_{j=0}^{t-1}\left(\begin{array}{c}
n-j k-i_{j}-1 \\
k-1
\end{array}\right)(j+1)^{i_{j+1}-i_{j}}
$$

is the number of initial segments, corresponding to the first stage (unbarred labels) of falling maximal chains of length $n-1-t(k-2)$ such that $i_{j+1}-i_{j}$ singletons are merged between the creation of the $(j+1)$-st and the $(j+2)$-nd $k$-block, for $j=0,1, \ldots, t-2$, and after the creation of the $t$-th $k$-block for $j=t-1$. This is so, because when creating the $(j+1)$-st $k$-block one must use the largest available singleton together with $k-1$ of the other $n-j k-i_{j}-1$ available singletons. Then for the following $i_{j+1}-i_{j}$ singleton merges one must use the $i_{j+1}-i_{j}$ largest currently available singletons in decreasing order. They must each be merged with one of the $j+1$ available non-singleton blocks. There are $(j+1)^{i_{j+1}-i_{j}}$ ways to carry out this process.

The second stage (barred labels) in the formation of a falling chain of length $n-1-t(k-2)$ starts from a partition having exactly $t$ blocks, all of them of size $\geq k$. Let these blocks be $B_{1}, B_{2}, \ldots, B_{t}$, ordered according to their maximal elements: $\max B_{1}<\ldots<\max B_{t}$. To maintain a falling label it is necessary to first merge $B_{t}$ with one of the $t-1$ earlier blocks, then merge this union with one of the remaining $t-2$ blocks, and so on. There are $(t-1)$ ! ways to carry this out.

We can get a simpler formula for the Betti numbers by counting the falling chains in another way. First we describe the falling chains in terms of standard tableaux of hook shape.

For $j \geq 0$, let $D_{k, 1^{j}}$ be the hook shaped Ferrers diagram with $k$ cells in the first row (which we call the arm) and one cell in each of the remaining $j$ rows (which we call the leg). For $j_{1}, j_{2}, \ldots, j_{t} \geq 0$ and $t \geq 1$, let $D^{k}\left(j_{1}, j_{2}, \ldots, j_{t}\right)$ be the skew diagram that consists of hook diagrams $D_{k, 1^{j_{i}}}, i=1,2, \ldots, t$, where the northeast corner of $D_{k, 1^{j_{i}}}$ is attached to the southwest corner of $D_{k, 1^{j_{i+1}}}$, for $i=1,2, \ldots, t-1$. We will refer to a skew diagram of this form as a broken hook diagram of type $(n, k, t)$, where $n$ is the number of cells, i.e. $n=k t+j_{1}+j_{2}+\ldots+j_{t}$. A tableau of broken hook shape $D$, is defined to be a filling of the cells of $D$ with distinct entries from $\{1,2, \ldots, n\}$. The tableau is said to be standard if the entries decrease along each row (from left to right) and along each column (from top to bottom). We shall say that a standard tableaux $T$ is left standard if $T$ also has the property that $n$ is in the leftmost possible cell of $D$, i.e. in the northwest corner cell 


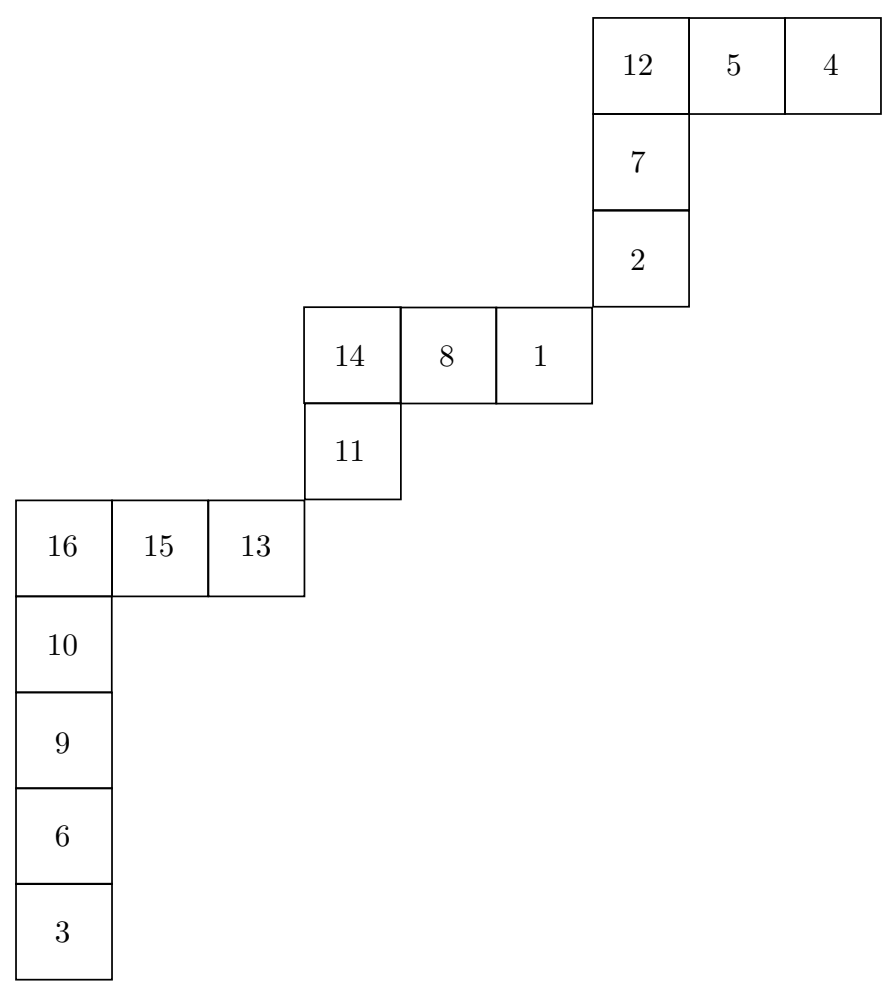

FIGURE 5

of the leftmost hook. For example, a left standard tableau of broken hook shape $D^{3}(4,1,2)$ is given in Figure 5 .

Let $T$ be a left standard tableau of broken hook shape $D=D^{k}\left(j_{1}, j_{2}, \ldots, j_{t}\right)$ and size $n$. We shall associate a falling chain $m_{T}$ with $T$. It has length $n-1-t(k-2)$ and is constructed from top to bottom in two stages (which are the reverse of the stages of the construction used in the proof of Corollary 6.2). Let $T_{i}$ be the hook tableau obtained by restricting $T$ to the $i$ th hook, $D_{k, 1^{j_{i}}}$. In the first stage we split off blocks consisting of the entries of $T_{i}$ from the remaining entries of $D^{k}\left(j_{1}, j_{2}, \ldots, j_{i-1}\right)$, in the order $i=t, t-1, \ldots, 2$. That is, the first partition below $\hat{1}$ has two blocks, one consisting of the entries of $T_{t}$. The next partition has three blocks, one consisting of the entries of $T_{t}$ and another consisting of the entries of $T_{t-1}$. At the end of the first stage we reach a partition with $t$ nonsingleton blocks corresponding to the hook tableaux $T_{1}, T_{2}, \ldots, T_{t}$.

In the second stage, we continue down the chain $m_{T}$ by either peeling a singleton from the bottom of the leg of one of the $T_{i}$ or by decomposing the arm of one of the $T_{i}$ into $k$ singletons after the leg has already been peeled away. At each step we choose either the smallest entry on the bottom of a leg or the arm with the smallest maximum, whichever is smaller.

It is easy to see that the maximal chain $m_{T}$ constructed above is falling, and that all falling chains can be uniquely obtained in this way. Indeed, the labels on the covering relations formed during the first stage of the construction are all 
equal to $\bar{n}$. The labels on the covering relations formed during the second stage are unbarred and are equal to the entry that is peeled away from the bottom of the leg or the maximum of the arm that is decomposed.

Recall from Section 4 that any maximal chain $m$ corresponds to a cocycle $\sigma^{m}$ which determines a cohomology class $\left[\sigma^{m}\right]$.

6.3. Corollary. For $1 \leq t \leq\left\lfloor\frac{n}{k}\right\rfloor$, let $\mathcal{T}_{n, k, t}$ be the set of all left standard tableaux with broken hook diagram of type $(n, k, t)$. Then the set $\left\{\left[\sigma^{m_{T}}\right] \mid T \in \mathcal{T}_{n, k, t}\right\}$ is a basis for the free Abelian group $\widetilde{H}^{n-3-t(k-2)}\left(\Delta_{n, k}\right)$. Consequently, the Betti numbers are given by

$$
\tilde{\beta}_{n, k}^{n-3-t(k-2)}=\sum_{\substack{j_{1}+j_{2}+\ldots+j_{t}=n \\
j_{i} \geq k}}\left(\begin{array}{c}
n-1 \\
j_{1}-1, j_{2}, \ldots, j_{t}
\end{array}\right) \prod_{i=1}^{t}\left(\begin{array}{c}
j_{i}-1 \\
k-1
\end{array}\right)
$$

Proof. The first statement follows from the correspondence between falling chains and left standard tableaux of broken hook shape, and from Theorems 4.3 and 5.9. Since the correspondence is one to one we can count left standard tableaux of broken hook shape to obtain the Betti numbers. The summand counts left standard tableaux of shape $D^{k}\left(j_{1}-k, j_{2}-k, \ldots, j_{t}-k\right)$. Indeed, the multinomial coefficient is the number of ways of distributing the numbers $1,2, \ldots, n-1$ among the $t$ tableaux. Once this has been done, $k-1$ numbers are selected for the arm of each tableaux. The binomial coefficients count the number of ways of making these selections.

The basis for cohomology given in Corollary 6.3 is used in [SW] to obtain an expression for the representation of the symmetric group acting on the cohomology of $\Delta_{n, k}$ in terms of a plethysm of the representation of the symmetric group on the cohomology of $\Delta\left(\overline{\Pi_{n}}\right)$ with irreducible representations of hook shape. There is also an explicit description of the dual basis for the homology of $\Delta_{n, k}$ in terms of left standard tableaux which will be discussed in a future paper.

\section{OTHER POSETS OF SET PARTITIONS}

The $k$-equal partition lattice can be characterized in several ways: via forbidden block sizes, as induced by the complete $k$-uniform hypergraph, or via its nature as intersection lattice for a highly symmetric subspace arrangement. It is natural to ask whether shellability can serve as a tool in the analysis of more general situations (other block sizes, other hypergraphs, other subspace arrangements). In this section we will provide some partial answers, positive as well as negative. For the connection with subspace arrangements see Section 3 of [B5].

Let $T$ be a set of positive integers containing $n$, and let

$$
\Pi_{n, T}=\left\{\pi \in \Pi_{n} \mid \text { all block sizes of } \pi \text { are in } T\right\} \text {. }
$$

If $1 \notin T$, we add the discrete partition to $\Pi_{n, T}$, so that $\Pi_{n, T}$ is a bounded poset in all cases. For $T=\{1, k, k+1, \ldots$,$\} this specializes to the k$-equal partition lattice $\Pi_{n, k}$ considered in the previous section. 
Here we will discuss the shellability of $\Pi_{n, T}$ for some other choices of $T$. In particular, the nonpure lattice

$$
\Pi_{n, k}^{\prime}=\Pi_{n,\{k, k+1, \ldots\}}
$$

will be analyzed from this point of view. $\Pi_{n, k}^{\prime}$ is the intersection lattice of a certain subspace arrangement embedded in the braid arrangement. For its analysis we will need a lemma about the CL-shellability of filters in geometric lattices.

A subset $A$ of a poset $P$ is called a filter if $x \geq y \in A$ implies that $x \in A$. If $P$ is bounded and $A \neq P$, we will consider $A \cup\{\hat{0}\}$, which is again a bounded poset. The atoms of $A \cup\{\hat{0}\}$ are the minimal elements of $A$.

A poset $P$ is totally semimodular if it is bounded and whenever two distinct elements $u$ and $v$ both cover $x$, and $u, v<y$, there exists $z \in P$ such that $z$ covers $u$ and $v$ and $z \leq y$. Examples are semimodular lattices, in particular geometric lattices.

7.1. Lemma. Let $A$ be a filter in a totally semimodular poset $P$. Then an ordering $a_{1}, a_{2}, \ldots, a_{t}$ of the minimal elements of $A$ is a recursive atom ordering of $A \cup\{\hat{0}\}$ if and only if property (ii) of Definition 5.10 holds.

Proof. The intervals $\left[a_{j}, \hat{1}\right]$ are totally semimodular, hence for them every atom ordering is recursive, by [BW2, Theorem 5.1]. For this reason property (i) of Definition 5.10 is superfluous.

We will apply this lemma and Theorem 5.11 to obtain the lexicographic shellability of $\Pi_{n, k}^{\prime}$, which (apart from $\hat{0}$ ) is a filter in the geometric lattice $\Pi_{n}$.

An atom of $\Pi_{n, k}^{\prime}$ is a partition $\pi$ such that $k \leq b<2 k$ for every block size $b$. Let $B_{1}, \ldots, B_{p}$ be the blocks of $\pi$ ordered by increasing order of minimum elements. Represent the atom $\pi$ by the $p$-tuple $w_{\pi}=\left(w_{1}, \ldots, w_{p}\right)$, where $w_{i}$ is the word obtained by listing the elements of $B_{i}$ increasingly. For example,

$$
\begin{aligned}
& w_{\pi}=(135,2679,4810), \\
& w_{\sigma}=(12368,457910)
\end{aligned}
$$

represent two atoms $\pi$ and $\sigma$ of $\Pi_{10,3}^{\prime}$.

The tuples $w_{\pi}$ can be ordered lexicographically, that is, both individual words $w_{i}$ and tuples of words $w_{\pi}$ are compared in lexicographic order. This induces a total order on the set of atoms of $\Pi_{n, k}^{\prime}$.

7.2. Theorem. The lexicographic order of the atoms of $\Pi_{n, k}^{\prime}$ is recursive. Consequently $\Pi_{n, k}^{\prime}$ is CL-shellable.

Proof. By Lemma 7.1 it suffices to verify the following:

$$
\text { if } w_{\sigma}<w_{\pi} \text { and } \sigma, \pi<\alpha \text { for two atoms } \sigma \text { and } \pi \text {, }
$$$$
\text { then there exists an atom } \tau \text { and an element } \beta
$$$$
\text { be covering } \pi \text { such that } w_{\tau}<w_{\pi} \text { and } \tau<\beta \leq \alpha \text {. }
$$

Let $\pi=B_{1}\left|B_{2}\right| \ldots \mid B_{p}$ and $\sigma=B_{1}^{\prime}\left|B_{2}^{\prime}\right| \ldots \mid B_{q}^{\prime}$, with blocks ordered by increasing minimal elements. Assume that $B_{j} \neq B_{j}^{\prime}$ and $B_{i}=B_{i}^{\prime}$ for $i<j$. Since $w_{\sigma}<w_{\pi}$, we must have that $w_{j}^{\prime}<w_{j}$, which leads to two cases. 
Case 1. $w_{j}^{\prime}$ is a prefix in $w_{j}$. Say that $w_{j}=w_{j}^{\prime} u$, the concatenation of words $w_{j}^{\prime}$ and $u \neq \emptyset$. Choose an element $x \in u$, and let $B_{f}^{\prime}, f>j$, be the block of $\sigma$ that contains $x$. Then $B_{j}^{\prime} \cup B_{f}^{\prime} \subseteq C$, where $C$ is the block of $\alpha$ that contains $B_{j}$, since $\pi, \sigma<\alpha$. Since $|u| \leq k-1$ and $\left|B_{f}^{\prime}\right| \geq k$, there exists some element $y \in B_{f}^{\prime}$ that is not in $u$. Let $B_{g}, g>j$, be the block of $\pi$ that contains $y$. Then $B_{j} \cup B_{g} \subseteq C$, so $\beta \leq \alpha$, where $\beta$ is the partition obtained from $\pi$ by merging $B_{j}$ and $B_{g}$. It remains to create an atom $\tau<\beta$ such that $w_{\tau}<w_{\pi}$. This is done by splitting the block $B_{j} \cup B_{g}$ of $\beta$ into one block $B_{j}^{\prime}$ and one or two other blocks that can be arbitrarily chosen.

Case 2. $w_{j}^{\prime}$ is not a prefix of $w_{j}$. Let $w_{j}^{\prime}=x_{1}^{\prime} x_{2}^{\prime} \ldots$ and $w_{j}=x_{1} x_{2} \ldots$, and let $s$ be minimal such that $x_{s} \neq x_{s}^{\prime}$. Then $x_{s}^{\prime}<x_{s}$ since $w_{j}^{\prime}<w_{j}$, and $s \geq 2$ by construction since $B_{i}=B_{i}^{\prime}$ for $i<j$. Let $B_{g}, g>j$, be the block of $\pi$ that contains $x_{s}^{\prime}$. Then $B_{j} \cup B_{g} \subseteq C$, where $C$ is the block of $\alpha$ that contains $B_{j}$. Let $\beta$ be the partition obtained from $\pi$ by merging $B_{j}$ and $B_{g}$. Then create an atom $\tau<\beta$ by splitting the new block of $\beta$ into $B_{j} \backslash\left\{x_{s}\right\} \cup\left\{x_{s}^{\prime}\right\}$ and $B_{g} \backslash\left\{x_{s}^{\prime}\right\} \cup\left\{x_{s}\right\}$. This satisfies condition $(*)$.

The result shows that the homology of $\Pi_{n, k}^{\prime}$ is torsion-free and gives (at least in principle) a method for computing its Betti numbers. We have not attempted to carry out this computation. A combinatorial description of the Betti numbers is derived in $[\mathrm{SaW}]$ by another method.

We have seen that the nonpure lattices $\Pi_{n, T}$ are shellable for $T=\{1, k, k+1, \ldots\}$ and $T=\{k, k+1, \ldots\}$. For $T=\{k, 2 k, 3 k, \ldots\}$ we get a pure lattice which was first shown to be CL-shellable by Wachs; see Sagan [Sa] and Wachs [W]. For $T=\{1, k+1,2 k+1, \ldots\}$ we get a pure poset which is totally semimodular and hence CL-shellable. This prompts the question: is $\Pi_{n, T}$ shellable for all choices $T$ of allowed block sizes? The answer is no.

7.3. Example. Let $T=\{3,5,6,9,10,12,15\}$. Then the proper part $\overline{\Pi_{15, T}}$ is the disjoint union of the connected pieces $\bar{\Pi}_{15,\{5,10,15\}}$ and $\bar{\Pi}_{15,\{3,6,9,12,15\}}$. Each such connected component is shellable, by the result of Wachs mentioned above, but their union is not, since it does not have the homotopy type of a wedge of spheres.

It doesn't help if $T$ is additive, meaning that $a, b \in T, a+b \leq n$ implies that $a+b \in T$. Let $T$ be as before and $T^{\prime}=\{3,5,6,8,9,10,11,12,13,14,15\}$. Then $T^{\prime}$ is additive but $\Pi_{15, T^{\prime}}=\Pi_{15, T}$, since the new allowed block sizes $8,11,13,14$ don't make any additional partitions legal. So, neither $\Pi_{15, T}$ nor $\Pi_{15, T^{\prime}}$ is shellable.

Another class of partition posets are the lattices $\Pi_{\lambda}$ defined in Björner [B5, $\S 3.3]$. Here $\lambda=\left(\lambda_{1}, \ldots, \lambda_{p}\right)$ is a partition of the integer $n, \lambda_{1} \geq \ldots \geq \lambda_{p}>0$, $\lambda_{1}+\ldots+\lambda_{p}=n$. The atoms of $\Pi_{\lambda}$ are the partitions of the set $[n]$ whose block sizes (decreasingly arranged) are $\lambda_{1}, \ldots, \lambda_{p}$, and $\Pi_{\lambda}$ consists of those partitions that are joins in $\Pi_{n}$ of collections of such atoms. For instance, for $\lambda=\left(k, 1^{n-k}\right)$ we get the $k$-equal lattice $\Pi_{n, k}$, and for $\lambda=\left(k^{n / k}\right)$ we get $\Pi_{n,\{k, 2 k, 3 k, \ldots\}}$. The $\Pi_{\lambda}$ 's are intersection lattices of certain subspace arrangements. We will now show that they are not shellable in general.

7.4. Example. Let $\lambda=(3,3,2,2,2)$, and let $\pi \in \Pi_{\lambda}$ be the coatom with blocks $B_{1}=\{1,2,3,4,5,6\}$ and $B_{2}=\{7,8,9,10,11,12\}$. If $\hat{0}<\sigma<\pi$ in $\Pi_{\lambda}$, then $B_{1}$ must be partitioned "mod $3 "$ in $\sigma$ and $B_{2}$ "mod 2 ", or vice versa. Hence, the open interval $(\hat{0}, x)$ is isomorphic to a disjoint union: 


$$
(\hat{0}, x) \cong\left(\widetilde{\Pi}_{\left(3^{2}\right)} \times \widetilde{\Pi}_{\left(2^{3}\right)} \backslash(\hat{1}, \hat{1})\right) \dot{\cup}\left(\widetilde{\Pi}_{\left(2^{3}\right)} \times \widetilde{\Pi}_{\left(3^{2}\right)} \backslash(\hat{1}, \hat{1})\right)
$$

where $\widetilde{\Pi}_{\lambda}=\Pi_{\lambda} \backslash\{\hat{0}\}$. Being the disjoint union of two posets of length 2 , $(\hat{0}, x)$ is not shellable, and hence so is not $\Pi_{\lambda}$.

A similar idea was used by Ziegler [Z1] to show that there exist disconnected intervals in the refinement poset of integer partitions.

By a hypergraph $\mathcal{H}$ we mean a collection of subsets of $[n]$. To avoid trivialities we will assume that $|A| \geq 2$ for all $A \in \mathcal{H}$, that $\bigcup_{A \in \mathcal{H}} A=[n]$, and that there are no proper inclusions among the members of $\mathcal{H}$. A hypergraph $\mathcal{H}$ is intersecting if $A \cap B \neq \emptyset$ for all $A, B \in \mathcal{H}$.

For subsets $A \subseteq[n]$ let $\pi_{A}$ be the partition of $[n]$ having $A$ as its only nonsingleton block, and let $\Pi_{\mathcal{H}}$ be the set of partitions obtainable as joins of elements $\pi_{A}, A \in \mathcal{H}$. Then $\Pi_{\mathcal{H}}$ is a lattice isomorphic to the intersection lattice of the hypergraph subspace arrangement $\mathcal{A}_{\mathcal{H}}$ introduced in [B5, § 3.1].

If $\mathcal{H}$ is the complete $k$-uniform hypergraph, we find that $\Pi_{\mathcal{H}}$ is the $k$-equal lattice $\Pi_{n, k}$, and if $\mathcal{H}$ is any 2-uniform hypergraph (i.e., "ordinary" graph), $\Pi_{\mathcal{H}}$ is a geometric lattice and hence EL-shellable. We will give an example of a 3 uniform hypergraph whose lattice $\Pi_{\mathcal{H}}$ is not shellable. The construction will use the simplicial complex $\mathcal{H}^{*}=\{B \subset[n] \mid B \cap A=\emptyset$ for some $A \in \mathcal{H}\}$ and the following technical fact.

7.5. Proposition. If the hypergraph $\mathcal{H}$ is intersecting, then $\bar{\Pi}_{\mathcal{H}}$ and $\mathcal{H}^{*}$ are homotopy equivalent.

The proof for this is based on the following lemma, which will be needed also later in the paper. For a proof of the lemma see Corollary 10.14 in [B6].

7.6. Lemma. Let $L$ be a finite lattice and $K$ the subset of elements $\wedge$-generated by its coatoms. Then $\bar{L}$ and $K \cap \bar{L}$ are homotopy equivalent.

Proof of Proposition 7.5. Since $\mathcal{H}$ is intersecting, we have that $\pi_{A} \vee \pi_{B}=\pi_{A \cup B}$. It follows that $\Pi_{\mathcal{H}}$ is isomorphic to the family of sets obtainable as unions of members of $\mathcal{H}$ and ordered by inclusion, a subposet of the Boolean algebra $2^{[n]}$. Passing to the set complements within $[n]$ we see that $\Pi_{\mathcal{H}}$ is antiisomorphic to the lattice $K$ of sets obtainable as intersections of facets of the complex $\mathcal{H}^{*}$. The facets of $\mathcal{H}^{*}$ are the coatoms of its face lattice $L$, and $\mathcal{H}^{*} \cong \bar{L}$ is a homeomorphism (barycentric subdivision). Now apply Lemma 7.6.

7.7. Example. Consider the intersecting hypergraph

$$
\mathcal{H}=\{123,125,136,145,146,234,246,256,345,356\}
$$

It can be checked that $\mathcal{H}^{*}$ triangulates the real projective plane. Hence, by Proposition 7.5 and Theorem 4.1, the lattice $\Pi_{\mathcal{H}}$ cannot be shellable.

\section{REFERENCES}

[BaG] K. Baclawski and A. Garsia, Combinatorial decompositions of a class of rings, Advances in Math. 39 (1981), 155-184. MR 84a:06003a

[Be] C. Berge, Principles of Combinatorics, Academic Press, New York, 1971. MR 42:5805 
[BiS] L.J. Billera and B. Sturmfels, Iterated fiber polytopes, Mathematika 41 (1994), 348-363. CMP 95:08

[B1] A. Björner, Shellable and Cohen-Macaulay partially ordered sets, Trans. Amer. Math. Soc. 260 (1980), 159-183. MR 81i:06001

[B2] Posets, regular CW complexes and Bruhat order, European J. Combinatorics 5 (1984), 7-16. MR 86e:06002

[B3] Some combinatorial and algebraic properties of Coxeter complexes and Tits buildings, Advances in Math. 52 (1984), 173-212. MR 85m:52003

[B4] The homology and shellability of matroids and geometric lattices, Matroid Applications (N. White, ed.), Cambridge Univ. Press, 1992, pp. 226-283. MR 94a:52030

[B5] - Subspace arrangements, First European Congress of Mathematics, Paris 1992 (A Joseph et al., eds.), Progress in Math., vol. 119, Birkhäuser, 1994, pp. 321-370.

[B6] Topological Methods, Handbook of Combinatorics (R. Graham, M. Grötschel and L. Lovász, eds.), North-Holland, 1995, pp. 1819-1872.

[B7] - Face numbers, Betti numbers and depth, in preparation.

[BK] A. Björner and G. Kalai, On $f$-vectors and homology, Combinatorial Mathematics: Proc. 3rd Intern. Conf., New York, 1985 (G. Bloom, R. Graham and J. Malkevitch, eds.), Annals of N. Y. Acad. Sci., vol. 555, New York Acad. Sci., 1989, pp. 63-80. MR 90i:52008

[B+] A. Björner, M. Las Vergnas, B. Sturmfels, N. White and G.M. Ziegler, Oriented Matroids, Cambridge Univ. Press, 1993. MR 95e:52023

[BL] A. Björner and L. Lovász, Linear decision trees, subspace arrangements and Möbius functions, Journal Amer. Math. Soc. 7 (1994), 677-706. MR 95e:52024

[BLY] A. Björner, L. Lovász and A. Yao, Linear decision trees: volume estimates and topological bounds, Proc. 24th ACM Symp. on Theory of Computing (May 1992), ACM Press, New York, 1992, pp. 170-177.

[BS] A. Björner and B. Sagan, Subspace arrangements of type $B_{n}$ and $D_{n}$, preprint 1994, J. Algebraic Combinatorics (to appear).

[BW1] A. Björner and M. Wachs, Bruhat order of Coxeter groups and shellability, Advances in Math. 43 (1982), 87-100. MR 83i:20043

[BW2] _ On lexicographically shellable posets, Trans. Amer. Math. Soc. 277 (1983), 323341. MR 84f:06004

[BW3] _ Generalized quotients in Coxeter groups, Trans. Amer. Math. Soc. 308 (1988), 1-37. MR 89c:05012

[BW4] _ Permutation statistics and linear extensions of posets, J. Combinatorial Theory, Ser. A 58 (1991), 85-114. MR 92m:06010

[BWa] A. Björner and J. Walker, A homotopy complementation formula for partially ordered sets, European J. Combinatorics 4 (1983), 11-19. MR 84f:06003

[BWe] A. Björner and V. Welker, The homology of " $k$-equal" manifolds and related partition lattices, Advances in Math. 110 (1995), 277-313.

[Bo] K. Bogart, The Möbius function of the domination lattice, unpublished manuscript, 1972.

[Br] T. Brylawski, The lattice of integer partitions, Discrete Math. 6 (1973), 201-219. MR 48:3752

[C] M.K. Chari, Steiner complexes, matroid ports and shellability, J. Combinatorial Theory, Ser. B 59 (1993), 41-68. MR 94e:05071

[Ga] A. Garsia, Combinatorial methods in the theory of Cohen-Macaulay rings, Advances in Math. 38 (1980), 229-266. MR 82f:06002

[G] C. Greene, A class of lattices with Möbius function $\pm 1,0$, European J. Combinatorics 9 (1988), 225-240. MR 89i:06012

[HT] S. Huang and D. Tamari, Problems of associativity: A simple proof for the lattice property of systems ordered by a semi-associative law, J. Combinatorial Theory, Ser. A 13 (1972), 7-13. MR 46:5191

[KK] B. Kind and P. Kleinschmidt, Schälbare Cohen-Macauley-Komplexe und ihre Parametrisierung, Math. Z. 167 (1979), 173-179. MR 80k:13010

[K] D.E. Knuth, Computer Musings: The associative law, or The anatomy of rotations in binary trees, Distinguished Lecture Series VII (Stanford, CA: University Video Communications, 1993), 68 minutes (videotape). 
[L1] S. Linusson, Partitions with restricted block sizes, Möbius functions and the $k$-of-each problem, preprint 1994.

[L2] - A class of lattices whose intervals are spherical or contractible, preprint, 1995.

[Mc] P. McMullen, The maximum numbers of faces of a convex polytope, Mathematika $\mathbf{1 7}$ (1970), 179-184. MR 44:921

[M] J.R. Munkres, Elements of Algebraic Topology, Addison-Wesley, Menlo Park, 1984. MR 85m:55001

[P1] J.M. Pallo, Enumerating, ranking and unranking binary trees, Computer J. 29 (1986), 171-175. CMP 18:13

[P2] Some properties of the rotation lattice of binary trees, Computer J. 31 (1988), 564-565. MR 89k:68122

[P3] An algorithm to compute the Möbius function of the rotation lattice of binary trees, Informatique Théor. et Applic./Theor. Informatics and Applic. 27 (1993), 341-348. MR 94i:68235

[PB] J.S. Provan and L.J. Billera, Decompositions of simplicial complexes related to diameters of convex polyhedra, Math. of Operations Research 5 (1980), 576-594. MR 82c:52010

[Q] D. Quillen, Homotopy properties of the poset of nontrivial p-subgroups of a group, Advances in Math. 28 (1978), 101-128. MR 80k:20049

[R] M. Rees, A basis theorem for polynomial modules, Proc. Cambridge Philos. Soc. 52 (1956), 12-16. MR 17:573i

[Sa] B. Sagan, Shellability of exponential structures, Order 3 (1986), 47-54. MR 87j:05020

$[\mathrm{SaW}]$ A. Sanders and M.L Wachs, The (co)homology of the lattice of partitions with lower bounded block size, in preparation.

[Sp] E.W. Spanier, Algebraic Topology, McGraw-Hill, New York, 1966. MR 35:1007

[S1] R.P. Stanley, Supersolvable lattices, Algebra Universalis 2 (1972), 197-217. MR 46:8920

[S2] _ Finite lattices and Jordan-Hölder sets, Algebra Universalis 4 (1974), 361-371. MR 50:6951

[S3] Cohen-Macaulay complexes, Higher Combinatorics (M. Aigner, ed.), NATO Advanced Study Institute Series, Reidel, Dordrecht/Boston, 1977, pp. 51-62. MR 58:28010

[S4] ㄴ. Balanced Cohen-Macaulay complexes, Trans. Amer. Math. Soc 249 (1979), 139157. MR 81c:05012

[S5] Combinatorics and Commutative Algebra, 2nd Ed. Progress in Mathematics, vol. 41, Birkhäuser, Boston, 1995. MR 85b:05002

[Su] S. Sundaram, Applications of the Hopf trace formula to computing homology representations, Proceedings of Jerusalem Combinatorics Conference, 1993 (H. Barcelo and G. Kalai, eds.), Contemporary Math., vol. 178, Amer. Math. Soc., 1994, pp. 277-309. CMP 95:06

[SW] S. Sundaram and M.L. Wachs, The homology representations of the $k$-equal partition lattice, preprint 1994.

[SWe] S. Sundaram and V. Welker, Group actions on arrangements of linear subspaces and applications to configuration spaces, preprint 1994.

[W] M.L. Wachs, A basis for the homology of the d-divisible partition lattice, preprint 1992, Advances in Math. (to appear).

[Wa] J.W. Walker, Canonical homeomorphisms of posets, Europ. J. Combinatorics 9 (1988), 97-107. MR 89g:06012

[We] V. Welker, Shellability in the lattice of subgroups of a finite group, Proceedings Jerusalem Combinatorics Conference, 1993 (H. Barcelo and G. Kalai, eds.), Contemporary Math., vol. 178, Amer. Math. Soc., Providence, RI, 1994, pp. 335-360. CMP 95:06

[YO] T. Yanagimoto and M. Okamoto, Partial orderings of permutations and monotonicity of a rank correlation statistic, Ann. Inst. Statist. Math. 21 (1969), 489-506. MR 41:2856

[Z1] G.M. Ziegler, On the poset of partitions of an integer, Journal of Combinatorial Theory, Ser. A 42 (1986), 215-222. MR 87k:06009

[Z2] _ Shellability of chessboard complexes, Israel J. Math. 87 (1994), 97-110. MR 95e:06010

Department of Mathematics, Royal Institute of Technology, S-100 44 Stockholm, SWEDEN

E-mail address: bjorner@math.kth.se

Department of Mathematics, University of Miami, Coral Gables, Florida 33124

E-mail address: wachs@math.miami.edu 